\title{
Theoretical Investigation of Thermodynamical and Structural Properties of 3d Liquid Transition Metals Using Different Reference Systems
}

\author{
Y. A. Sonvane, ${ }^{1}$ P. B. Thakor, ${ }^{2}$ and A. R. Jani ${ }^{3}$ \\ ${ }^{1}$ Department of Applied Physics, S. V. National Institute of Technology, Gujarat, Surat 395 007, India \\ ${ }^{2}$ Department of Physics, Veer Narmad South Gujarat University, Gujarat, Surat 395 007, India \\ ${ }^{3}$ Department of Physics, Sardar Patel University, Gujarat, Vallabh Vidyanagar 388 120, India
}

Correspondence should be addressed to Y. A. Sonvane, yas@ashd.svnit.ac.in

Received 16 September 2012; Accepted 5 October 2012

Academic Editors: A. Ananikian, M. Appell, and M. Sanati

Copyright () 2012 Y. A. Sonvane et al. This is an open access article distributed under the Creative Commons Attribution License, which permits unrestricted use, distribution, and reproduction in any medium, provided the original work is properly cited.

\begin{abstract}
The present paper deals with the theoretical investigation of thermodynamical and structural properties like internal energy $(E)$, entropy $(S)$, Helmholtz free energy $(F)$, isothermal compressibility $\left(\chi_{T}\right)$, specific Heat $\left(C_{V}\right)$, structure factor $S(q)$, and long wave length limit $S(0)$ of structure factor of $3 \mathrm{~d}$ liquid transition metals. To describe electron-ion interaction we have used our newly constructed parameter free model potential. To perform this task, we have used different reference systems like Percus Yevick Hard Sphere (PYHS), One Component Plasma (OCP), and Charged Hard Sphere (CHS) reference systems. We have also seen the influence of different local field correction functions like Hartree (HR), Taylor (TR), and Sarkar et al. (SR) on thermodynamical properties of $3 \mathrm{~d}$ liquid transition metals. Finally we conclude that the proper choice of the model potential along with reference system plays a vital role in the study of thermodynamical and structural properties of $3 \mathrm{~d}$ liquid transition metals.
\end{abstract}

\section{Introduction}

The structure and thermodynamics of liquid metals have been widely studied with an increasing sophistication in the modelling of the interionic forces and in the classical statistical mechanics treatment of ionic correlations [1-48]. This study has drawn much theoretical attention both for its intrinsic interest and for the relevance to an understanding of electronic properties. The calculations of the atomic interactions in bonded metals have mainly been based on the density dependent pairwise potentials derived from electronion pseudopotential with the linear response theory and second order perturbation theory. The statistical mechanics side of the problem has been calculated with perturbation theory integral equations and computer simulations [1-10]. Although a lot of work has been done on both the structure and thermodynamics, still some questions await a definite answer. The limitations of linear response theory based interactions are well known. It is also possible to consider the interactions based on second order perturbation theory as effective pairwise potentials [11, 12]. However, in such case one would expect that a combined study of the structural and thermodynamic consequences of the interaction could illuminate in a quantitative way the relevance of such effective model interactions for the explanation of the physical and chemical properties of the liquid metals. We believe that only a combined analysis of structural and thermodynamic data should be able to assess the quality of any model for the interionic forces. However, only a few studies have been made addressing simultaneously these two problems.

In all these attempts the use of pseudopotential approach is found a remarkable success. But many existing studies have been limited to local model potentials with empirically determined parameters [13-15]. Also it is found in our literature survey [1-50] that some abinitio pseudopotentials suitable for the perturbation theory of the ionic interactions were generated. In general, the pseudopotentials like Ashcroft empty core model (AS) [16-19], Heine-Abarenkov 
model (HA) [17, 20, 21], harmonic model potential (HMP) $[17,20,21]$, generalized nonlocal model potential (GNMP) [22-24], energy independent nonlocal model potential (EINMP) $[25,26]$ and so forth. have been applied to study the structure and thermodynamics of liquids.

On the other side of the treatment of the atomic correlations, computer simulation has a precision limited only by numerical errors. However, when focusing on trends of thermodynamic quantities, approximate but reliable theories aimed at modelling the free energy are still very useful tools. The simplest of such method is Gibbs-Bogoliubov (GB) approach $[24,27,28]$.

The general idea underlying the GB $[24,27,28]$ inequality is that the true Helmholtz free energy of a liquid metal is bounded from above by the free energy of a suitably chosen reference system plus the difference in average potential energy between the actual and the reference system, calculated using the distribution functions of the reference systems. Thus the choice of the reference system is clearly very important. Ideally the basis for choosing the best reference system is that one expects it to give the lowest free energy and the expressions required for the thermodynamic and structure can be expressed possibly in an analytic form. People have applied popular reference system like Percus-Yevick Hard Sphere (PYHS) [23, 2938], One Component Plasma (OCP) [37-44], Charged Hard Sphere (CHS) [29, 30, 45-48], Hard Sphere Yukawa (HSY) $[49,50]$, Optimized Random Phase Approximation (ORPA) [24], and Soft Sphere (SS) systems [23].

Thus it is highly desirable to search a better reference system which gives good explanation of various properties when it is used with a particular model potential. Hence proper combination of a model potential and a reference system is one of the basic requirement in explaining various structure and thermodynamics of liquid metals. So, we thought it worthwhile to study the structure and thermodynamics of liquid transition metals using our own model potential using different reference systems.

In the last two decades, the considerable efforts have been made to understand the structure and thermodynamics of several simple liquid metals, liquid transition metals, liquid rare-earth metals, and their alloys [1-50]. The interatomic potentials of simple metals [24, 37-39, 45-49, 51-55] have been fully investigated and their thermodynamic properties could be derived with sufficient accuracy, but in the case of transition metals the hybridization of $d$ electron with $s$ electron makes the things complex. Computer experiments are always intended to propose a plausible interpretation of experimental results in some cases to give the solution to an experimentally inaccessible problem. Despite the success of the theory in the solid state, results for the structure factor of liquid transition metals using molecular dynamics and other complicated liquid state theories have not been so reliable $[1-10]$. The reliability of the predicted values, however, entirely depends on the validity of a given interatomic potential and the model used. Compare to simple liquid metals only a few attempts are made on liquid transition metals [2-10, 50, 55-58]. Therefore, the present paper deals with the computation of thermodynamical and structural properties like internal energy $(E)$, entropy $(S)$, Helmholtz free energy $(F)$, isothermal compressibility $\left(\chi_{T}\right)$, specific heat $\left(C_{V}\right)$, structure factor $S(q)$, and long wave length limit of structure factor $S(0)$ of $3 \mathrm{~d}$ liquid transition metals using our newly constructed parameter free model potential [43, 59, $60]$ with different reference systems like Percus-Yevick Hard Sphere (PYHS) [23, 29-38], One Component Plasma (OCP) [37-44], and Charged Hard Sphere (CHS) [29, 30, 45-48] systems.

An important application of pseudopotential is the calculation of structural properties of disordered materials such as liquid, amorphous, and their alloys. The problem with model pseudopotential is that of their transferability, because still there is no accurate method to obtain the form factor by which all the properties of liquid metals may be successfully investigated. It is found that a particular pseudopotential may be suitable for some properties of some metals and unsuitable for other properties of other metals. The usefulness of any model potential depends on how many number of parameters it involves. Generally, the potential involving less number of parameters is considered to be, comparatively a better one than that involving more number of parameters because it avoids more complexities in the calculation. It has been observed that a unique method of the determination of the potential parameter has not been pointed out so far. A number of investigators have used fitting procedure in which the potential parameters are fitted in such a way that a good agreement with experimental findings is to be obtained. Such a procedure will generally give good results for a certain property, but the same set of parameters will not give good results for other properties. Hence, we thought it worthwhile to construct a parameter free model potential which, by employing, explains the physical as well as chemical properties of condensed matter.

A pseudopotential method employing a simple model of a solid composed of atomic cores and valence electrons can predict the existence and properties of new solids and their properties [61]. By implementing this idea we have constructed a new model potential which is splitted into three regions $[43,59,60]$,

$$
W_{B}(r)= \begin{cases}0, & r<r_{i}, \\ -\left(\frac{Z e^{2}}{r_{a}}\right)\left\{2-\exp \left(\frac{r}{r_{a}}-1\right)\right\}, & r_{i} \leq r \leq r_{a}, \\ -\left(\frac{Z e^{2}}{r}\right), & r>r_{a} .\end{cases}
$$

Basically, this is the modified version of the Ashcroft's empty core model [62]. Here we have considered actual core of an ion as an empty core rather than pseudizing it. The whole effective region is splitted into three parts. The effective weak potential felt by an electron is sandwiched between empty core and long range coulomb potential which is continuous at $r=r_{a}$ and is considered between the ionic radius $\left(r_{i}\right)$ and atomic radius $\left(r_{a}\right)$.

In the reciprocal space, the corresponding bare-ion form factor of the present model potential is given by $[43,59,60]$ 


$$
W_{B}(q)=\frac{-4 \pi Z e^{2}}{q^{3} r_{a \Omega}\left(1+q^{2} r_{a}^{2}\right)^{2}}\left[\begin{array}{c}
2\left(1+2 q^{2} r_{a}^{2}\right) \sin \left(q r_{a}\right)-q r_{a}\left(1+3 q^{2} r_{a}^{2}\right) \cos \left(q r_{a}\right)+2\left(1+q^{2} r_{a}^{2}\right)^{2} \\
\times\left\{q r_{i} \cos \left(q r_{i}\right)-\sin \left(q r_{i}\right)\right\}-\exp \left(\frac{r_{i}}{r_{a}}-1\right) \\
\times\left\{\begin{array}{l}
\left(-2 q^{3} r_{a}^{3}+q^{3} r_{a}^{2} r_{i}+q^{5} r_{a}^{4} r_{i}\right) \cos \left(q r_{i}\right) \\
-\left(-q^{2} r_{a}^{2}+q^{4} r_{a}^{4}+q^{4} r_{a}^{3} r_{i}+q^{2} r_{a} r_{i}\right) \sin \left(q r_{i}\right)
\end{array}\right\}
\end{array}\right] .
$$

Here $Z, e, \Omega, q, r_{i}$, and $r_{a}$ are the valency, electronic charge, atomic volume, wave vector, ionic radius, and atomic radius, respectively. The local field correction functions like Hartree (HR) [63], Taylor (TR) [64], and Sarkar et al. (SR) [65] have been applied to see the influence of exchange and correlation effect on thermodynamical properties of liquid transition metals.

\section{Theory}

The Helmholtz free energy $(F)$ lies at the heart of the pseudopotential perturbation scheme to calculate the thermodynamical properties of liquid metals. The Helmholtz free energy of the system is defined by the use of the GB inequality as follow:

$$
F \leq F_{0}+\left\langle H-H^{0}\right\rangle,
$$

where $F_{0}$ is the Helmholtz free energy (per ion) of the reference system, $H$ and $H^{0}$ denote the Hamiltonians of the real and reference system, respectively.

Again,

$$
F=E-T S
$$

with $E, S$, and $T$ are internal energy, entropy, and absolute temperature respectively.

The internal energy of reference system is given by

$$
E=\frac{3}{2} k_{B} T+F_{1}+F_{\mathrm{EG}}+F_{\mathrm{BS}}+F_{M}
$$

$F_{1}$ is the first order electronic term arising from the average interaction of the valence electron with noncoulombic part of the bare ion pseudopotential. One has

$$
F_{1}=\lim _{q \rightarrow 0}\left\{W_{B}(q)+\frac{4 \pi Z}{q^{2}}\right\} \frac{Z}{\Omega} .
$$

$F_{\mathrm{EG}}$ is the free energy of the electron gas. One has

$$
\begin{aligned}
F_{\mathrm{EG}}=N Z\left\{\frac{3}{10} k_{F}^{2}-\frac{3}{4 \pi} k_{F}-0.0474\right. \\
\left.\quad-0.0155 \ln k_{F}-\frac{1}{12} k_{B}^{2} T^{2} N\left(E_{F}\right)\right\} .
\end{aligned}
$$

Here $N\left(E_{F}\right)$ is the density of the states per unit volume at Fermi energy. $F_{\mathrm{BS}}$ is the band structure energy and is given in terms of $F_{N}(q)$, the normalized energy wave number characteristic incorporating exchange and correlation corrections as,

$$
F_{\mathrm{BS}}=-\frac{z^{2}}{\pi} \int_{0}^{\infty} S(q) F_{N}(q) d q
$$

Here $S(q)$ is the structure factor of reference system. $F_{N}(q)$ is the normalized energy wave number characteristic. It is expressed as

$$
F_{N}(q)=\left(\frac{q^{2}}{4 \pi Z^{2} e^{2}}\right)^{2}\left[1-\frac{1}{\varepsilon(q)}\right]\left[\frac{1}{1-f(q)}\right]\left|W_{B}(q)\right|^{2} .
$$

Here $\varepsilon(q)$ is called the modified dielectric function. It includes exchange and correlation effect in the screening through local field correction function $f(q) . W_{B}(q)$ is the unscreened form factor. It depends on the core-ion pseudopotential and hence allows us to investigate its effect on the thermodynamics function. Here $\varepsilon(q)$ is the modified Hartree dielectric screening function which takes into account the conduction electron interaction

$$
\mathcal{\varepsilon}(q)=1+\left[1+\varepsilon_{H}(q)\right][1-f(q)]
$$

$\varepsilon_{H}(q)$ is the static dielectric function and $f(q)$ is the correction factor for the exchange and correlated motion of the conduction electrons. $F_{M}$ is the Madelung energy and it can be written as follows.

For PYHS systems

$$
F_{M}=\frac{Z^{2} \pi \sigma^{2}}{(1+2 \eta) \Omega}\left(1-\frac{\eta}{5}+\frac{\eta^{2}}{10}\right) \text {. }
$$

For OCP system

$$
F_{M}=k_{B} t\left(\frac{\Gamma^{*}-\Gamma}{\Gamma}\right)\left(A \Gamma+B \Gamma^{1 / 4}+B \Gamma^{-1 / 4}+D\right) .
$$

The constants $A, B, C$, and $D$ are $-0.89752,0.94544,0.17954$, and -0.80049 , respectively. $\Gamma^{*}$ is effective plasma parameter, $\Gamma^{*}=Z^{2} e^{2} \beta / r_{s}, r_{s}$ is the radius of the atomic sphere, $r_{s}=$ $(3 \Omega / 4 \pi)^{3} \cdot \beta=k_{B} t$, where $k_{B}$ and $t$ are Boltzmann constant and absolute temperature, respectively.

For CHS systems

$$
\begin{aligned}
F_{M}= & k_{B} t\left(\frac{\Gamma^{*}-\Gamma}{2 \eta^{1 / 3}}\right) \\
& \times\left(1+\eta-\frac{\eta^{2}}{5}-\frac{1+2 \eta}{k}\{\sqrt{1+2 k \xi}-1\}\right),
\end{aligned}
$$

where $\zeta=(1-\eta)^{3} /(1+2 \eta)^{2}$ and $k=\left(12 \eta^{2 / 3} \Gamma\right)^{1 / 2}$. 
TABLE 1: The input parameters used in the present computations.

\begin{tabular}{lcccc}
\hline Metal & $Z$ & $T(\mathrm{~K})[65,66]$ & Density $n\left(\mathrm{gm} / \mathrm{cm}^{3}\right)[65,66]$ & $r_{i}(\AA)$ \\
\hline Sc & 1.50 & 1833 & 2.92 & 0.81 \\
$\mathrm{Ti}$ & 1.50 & 1973 & 4.15 & 0.68 \\
$\mathrm{~V}$ & 1.50 & 2173 & 5.36 & 0.59 \\
$\mathrm{Cr}$ & 1.50 & 2173 & 6.26 & 0.64 \\
$\mathrm{Mn}$ & 1.50 & 1533 & 5.96 & 0.80 \\
$\mathrm{Fe}$ & 1.50 & 1823 & 7.00 & 1.45 \\
$\mathrm{Co}$ & 1.50 & 1823 & 7.71 & 0.64 \\
$\mathrm{Ni}$ & 1.50 & 1773 & 7.71 & 0.74 \\
$\mathrm{Cu}$ & 1.50 & 1423 & 7.96 & 1.37 \\
\hline
\end{tabular}

The computation of the entropy is more straight forward and computed using $S=S_{\text {gas }}+S_{\text {elec }}+S_{i}$ with,

$$
S_{\text {gas }}=\frac{5}{2} k_{B}+k_{B} \ln \left\{\Omega\left(\frac{k_{B} T}{4 \pi}\right)^{1 / 2}\right\}, \quad S_{\text {elec }}=\left(\frac{\pi^{2} k_{B}^{2} T}{k_{F}^{2}}\right),
$$

where $S_{i}$ term represents the contribution from the different reference system.

For PYHS system

$$
S_{\mathrm{PYHS}}=\frac{k_{B} \eta(3 \eta-4)}{(1-\eta)^{2}} .
$$

For OCP system

$$
\begin{aligned}
S_{\mathrm{OCP}}= & -3 B \Gamma^{1 / 4}+5 C \Gamma^{-1 / 4}-D \ln \Gamma \\
& +D[A+4(B-C)]+0.420 .
\end{aligned}
$$

For CHS system

$$
\begin{aligned}
S_{\mathrm{CHS}}= & \frac{(1+2 \eta) k_{B}}{72 \xi \eta} \\
& \times\left\{3 k \xi[\sqrt{1+2 k \zeta}+1]-2\left[(1+2 k \zeta)^{3 / 2}-1\right]\right\} \\
& -k_{B}\left[\frac{6 \eta}{1-\eta}+2 \ln (1-\eta)\right] .
\end{aligned}
$$

For a given number density $\rho$, packing fraction $\eta$ (HS, CHS, and SS reference systems) and plasma parameter $\Gamma$ (OCP reference system) are varied to obtain a minimum variational upper bound for the Helmholtz free energy $(F)$.

The long wave length limit of structure factor $S(q)$ is defined as follows.

For PYHS

$$
S(0)=\frac{(1-\eta)^{4}}{(1+2 \eta)^{2}}
$$

For OCP system

$$
\begin{aligned}
S(0)= & \lim _{q \rightarrow 0} S(q) \\
= & {\left[\begin{array}{l}
1-\frac{\Gamma}{20 \alpha_{2}^{2}}\left(5 \alpha_{2}^{4}+\alpha_{1}^{4}\right) \\
+4 \pi Z^{2} e^{2} \beta \rho\left\{\begin{array}{c}
\frac{5}{3} r_{a}^{2}+\frac{4}{3} \frac{r_{i}^{3}}{r_{a}} \\
-2 \exp \left(\frac{r_{i}}{r_{a}}-1\right) \\
\times\left(2 r_{a}^{2}-2 r_{a} r_{i}+r_{i}^{2}\right) \\
+\frac{\pi}{4 k_{F}}-\frac{\gamma_{0}}{k_{F}^{2}}
\end{array}\right\}
\end{array}\right]^{-1} . }
\end{aligned}
$$

For CHS system

$$
S(0)=\lim _{q \rightarrow 0} S(q)
$$

$$
=\left[4 \pi \rho a \Gamma\left\{\begin{array}{l}
\frac{5}{3} r_{a}^{2}+\frac{4}{3} \frac{r_{i}^{3}}{r_{a}} \\
-2 \exp \left(\frac{r_{i}}{r_{a}}-1\right) \\
\times\left(2 r_{a}^{2}-2 r_{a} r_{i}+r_{i}^{2}\right) \\
-\frac{\sigma^{2}(\alpha-1)}{12 \eta^{2 / 3} \Gamma}+\frac{\pi}{4 k_{F}}+\frac{\gamma_{0}}{k_{F}^{2}}
\end{array}\right]\right]^{-1} .
$$

The isothermal compressibility $\chi_{T}$ and specific heat $C_{V}$ at constant volume can be expressed as

$$
\chi_{T}=\frac{S(0)}{\rho k_{B} T}, \quad \frac{C_{V}}{k_{B}}=\frac{t}{k_{B}} \frac{\partial S}{\partial t} .
$$

\section{Results and Discussion}

The thermodynamical variational properties of $3 \mathrm{~d}$ series of liquid transition metals elements have been investigated without any adjustable parameter. Table 1 represents the input parameters used in the present computation. Selfconsistent calculations by Moriarty [73] indicate that elements whose properties are affected by $\mathrm{d}$ bands, the valency 
TABLE 2: Calculated variational parameter (packing fraction $\eta$ and plasma parameter $\Gamma$ ) due to different local field correction functions like Hartree (HR) [63], Taylor (TR) [64], and Sarkar et al. (SR) [65] of 3d liquid transition metals.

\begin{tabular}{|c|c|c|c|c|c|c|c|c|c|c|}
\hline \multirow{2}{*}{ Metal } & \multirow{2}{*}{$S(q)$} & \multicolumn{5}{|c|}{ Packing fraction $(\eta)$} & \multicolumn{4}{|c|}{ Plasma parameter $(\Gamma)$} \\
\hline & & HR [63] & TR [64] & SR [65] & Others $[34,45,48,50]$ & Expt. [67] & $\operatorname{HR}[63]$ & TR [64] & SR [65] & Others $[44,45,56]$ \\
\hline \multirow{2}{*}{ Sc } & PYHS & 0.4395 & 0.4295 & 0.4292 & \multirow{2}{*}{$0.353,0.330,0.325,0.430$} & \multirow{2}{*}{0.43} & \multirow{2}{*}{66} & \multirow{2}{*}{68} & \multirow{2}{*}{68} & \multirow{2}{*}{ - } \\
\hline & CHS & 0.4302 & 0.4294 & 0.4299 & & & & & & \\
\hline \multirow{2}{*}{$\mathrm{Ti}$} & PYHS & 0.4323 & 0.4317 & 0.4314 & \multirow{2}{*}{$0.415,0.415,0.418,0.362$} & \multirow{2}{*}{0.44} & \multirow{2}{*}{70} & \multirow{2}{*}{72} & \multirow{2}{*}{72} & \multirow{2}{*}{110,105} \\
\hline & CHS & 0.4330 & 0.4348 & 0.4337 & & & & & & \\
\hline \multirow{2}{*}{$\mathrm{V}$} & PYHS & 0.4442 & 0.4338 & 0.4335 & \multirow{2}{*}{$0.418,0.420,0.425,0.374$} & \multirow{2}{*}{0.44} & \multirow{2}{*}{64} & \multirow{2}{*}{64} & \multirow{2}{*}{64} & \multirow{2}{*}{110,105} \\
\hline & CHS & 0.4312 & 0.4291 & 0.4316 & & & & & & \\
\hline \multirow{2}{*}{$\mathrm{Cr}$} & PYHS & 0.4406 & 0.4389 & 0.4394 & \multirow{2}{*}{$0.443,0.445,0.445,0.380$} & \multirow{2}{*}{0.45} & \multirow{2}{*}{68} & \multirow{2}{*}{68} & \multirow{2}{*}{66} & \multirow{2}{*}{110,105} \\
\hline & CHS & 0.4347 & 0.4365 & 0.4349 & & & & & & \\
\hline \multirow{2}{*}{$\mathrm{Mn}$} & PYHS & 0.4662 & 0.4665 & 0.4658 & \multirow{2}{*}{$0.500,0.508,0.515,0.404$} & 0.45 & 96 & 96 & 96 & 110,105 \\
\hline & CHS & 0.4648 & 0.4619 & 0.4630 & & & & & & \\
\hline $\mathrm{Fe}$ & PYHS & 0.4551 & 0.4549 & 0.4553 & $0.435,0.430,0.389,0.411$ & 0.44 & 86 & 86 & 88 & 110,105 \\
\hline & CHS & 0.4585 & 0.4581 & 0.4569 & & & & & & \\
\hline $\mathrm{Co}$ & PYHS & 0.4547 & 0.4548 & 0.4540 & $0.420,0.405,0.400,0.370$ & 0.45 & 74 & 74 & 72 & 110,105 \\
\hline & CHS & 0.4582 & 0.4606 & 0.4630 & & & & & & \\
\hline $\mathrm{Ni}$ & PYHS & 0.4572 & 0.4562 & 0.4566 & $0.405,0.380,0.373,0.351$ & 0.45 & 88 & 88 & 88 & 110,105 \\
\hline & CHS & 0.4614 & 0.4631 & 0.4619 & & & & & & \\
\hline $\mathrm{Cu}$ & PYHS & 0.4764 & 0.4766 & 0.4758 & $0.418,0.383,0.370,0.450$ & 0.46 & 106 & 106 & 106 & - \\
\hline & CHS & 0.4842 & 0.4840 & 0.4845 & & & & & & \\
\hline
\end{tabular}

(Z) lie in a narrow range $1.1<Z<1.7$. By implementing this concept, we have taken $Z=1.5$ for $3 \mathrm{~d}$ liquid transition metals, respectively. Therefore the number of $\mathrm{d}$ electrons, which do not participate to conduction, is fixed since $Z_{s}+$ $Z_{d}$ is constant. We have displaced in Table 2 the values of the variational parameter with Hartree (HR) [63], Taylor (TR) [64] and Sarkar et al. (SR) [65] local field correction functions using Percus-Yevick Hard Sphere (PYHS) [23, 2938], One Component Plasma (OCP) [37-44], and Charged Hard Sphere (CHS) [29, 30, 45-48] reference systems for 3d liquid transition metals. The present results of variational parameter $\eta$ are quite good in agreement with experimental data [67] as well as other theoretical data [36, 45, 48, 50] whereas in the case of plasma parameter $(\Gamma)$ the experimental data is not available, but with other theoretical data [44, $45,56]$ it is reasonable because we have taken actual plasma parameter $(\Gamma)$ rather than fitting it as done by others [45]. We have noticed that the effect of local field correction function on variational parameters $(\eta$ and $\Gamma$ ) is very small. It is also found that the largest values of variational parameter $(\eta$ and $\Gamma$ ) are obtained when the $d$ band is filled to a large extent, while lowest values are obtained when the $\mathrm{d}$ band is nearly empty in the case of $3 \mathrm{~d}$ liquid transition metal. This is clearly related to the strongest bonding which occurs when only the bonding but not the antibonding states are occupied. Figure 1 represents the variational parameters $(\eta$ and $\Gamma$ ) with Hartree (HR) [63] local field correction for 3d liquid transition metals using different reference systems.

Figure 2 represents the Helmholtz free energy versus variational parameter of $\mathrm{Cu}$ liquid transition metals due

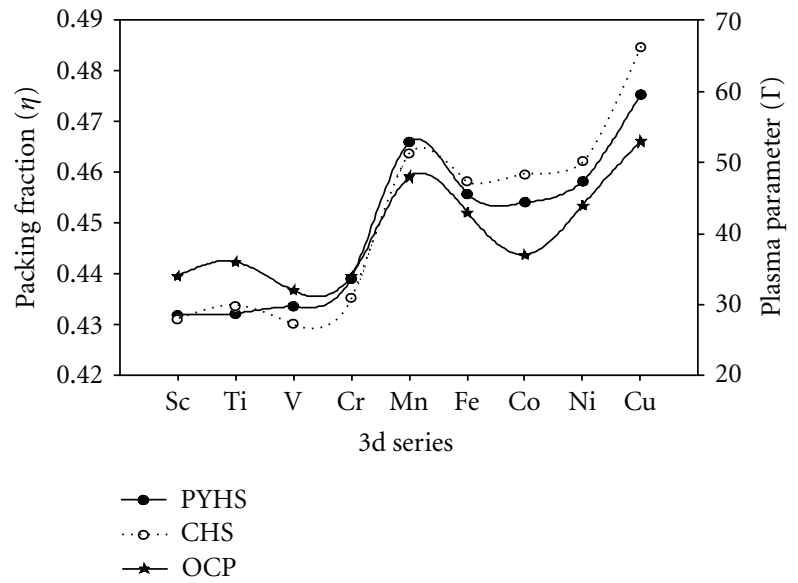

FIGURE 1: Packing fraction $(\eta)$ and plasma parameter $(\Gamma)$ obtained from the variational method for $3 \mathrm{~d}$ liquid transition metals.

to Sarkar et al. (SR) [65] local field correction function. The OCP system gives the lowest Helmholtz free energy compared to other reference systems. Figure 2 represents Helmholtz free energy versus variational parameter of liquid $\mathrm{Cu}$ for different local field correction function due to CHS system, which indicates that Taylor (TR) [64] local field correction function gives the lowest Helmholtz free energy, while Hartree (HR) [63] gives maximum Helmholtz free energy. 


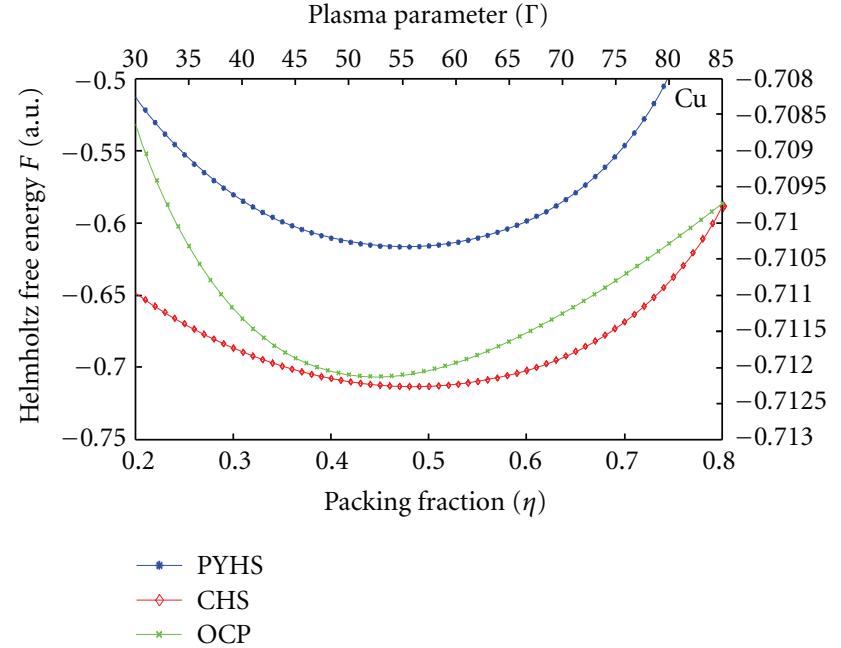

(a)

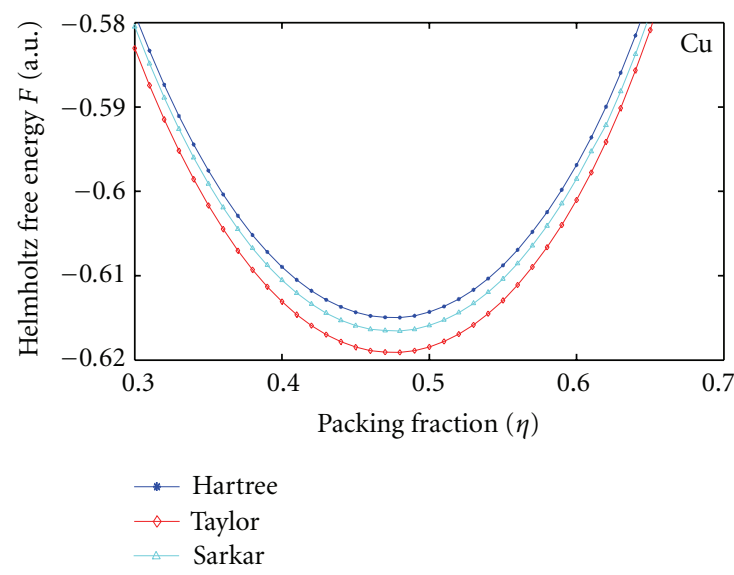

(b)

FIGURE 2: Helmhotz free energy versus variational parameters (packing fraction $(\eta)$ and Plasma parameter $(\Gamma)$ ) for different reference systems due to Sarkar et al. (SR) [65] local field correction function and for different local field correction function due to CHS system of Cu liquid metals.

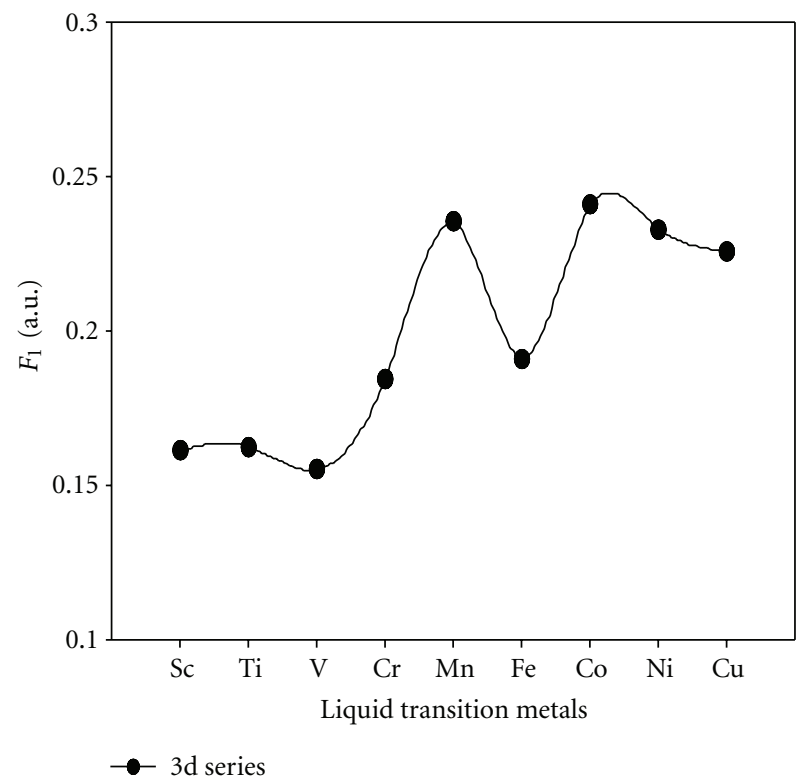

(a)

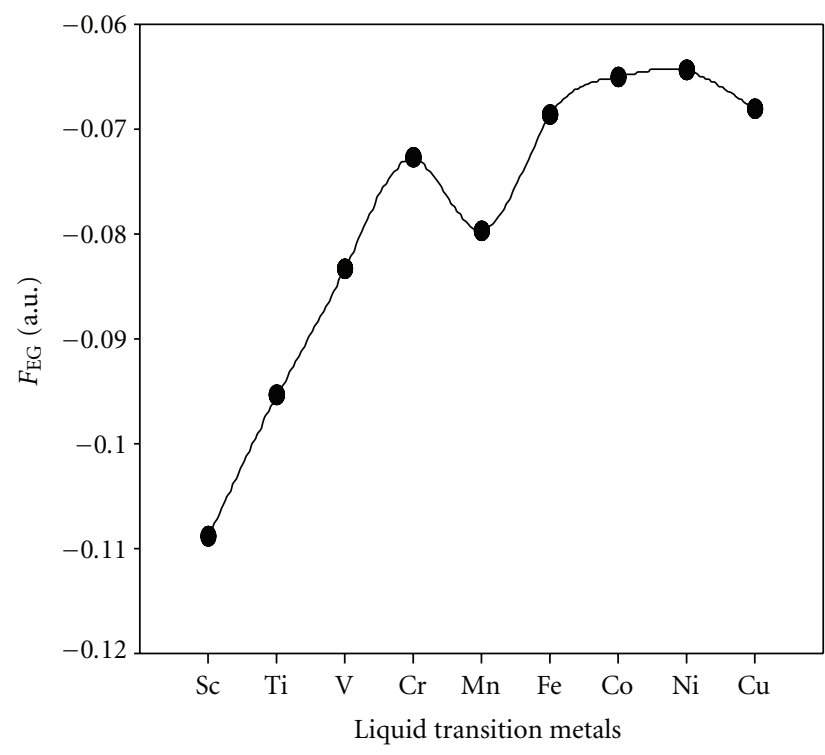

$3 \mathrm{~d}$ series

(b)

FIgURe 3: Calculated first order electronic energy $\left(F_{1}\right)$ and free energy of the electron gas $\left(F_{\mathrm{EG}}\right)$ of liquid transition metals.

Figure 3 shows calculated $F_{1}$ and $F_{\mathrm{EG}}$ terms of $3 \mathrm{~d}$ liquid transition metals. $F_{1}$ and $F_{\mathrm{EG}}$ terms are independent of the reference system and influence of local field correction function so that they remain constant for each system. Figure 4 represents Band structure energy $F_{\mathrm{BS}}$ and the effect of local field correction on $F_{\mathrm{BS}}$ term of $3 \mathrm{~d}$ liquid transition metals. $F_{\mathrm{BS}}$ depends upon both reference system and local field correction functions. The OCP system gives the lowest value of Band structure energy. The maximum value of $F_{\mathrm{BS}}$ is obtained for $3 \mathrm{~d}$ series for liquid Co. The deviation of local field correction on $F_{B}$ is going on increasing from $\mathrm{Sc}$ to $\mathrm{Cu}$ as the $\mathrm{d}$ shell is going to fill up (or atomic number is going on increasing). The Taylor (TR) [64] shows less deviation of $F_{\mathrm{BS}}$ while Sarkar et al. (SR) [65] shows much deviation from Hartree (HR) [63] for CHS system. Figure 5 represents calculated value of Madelung energy $F_{M}$ term 


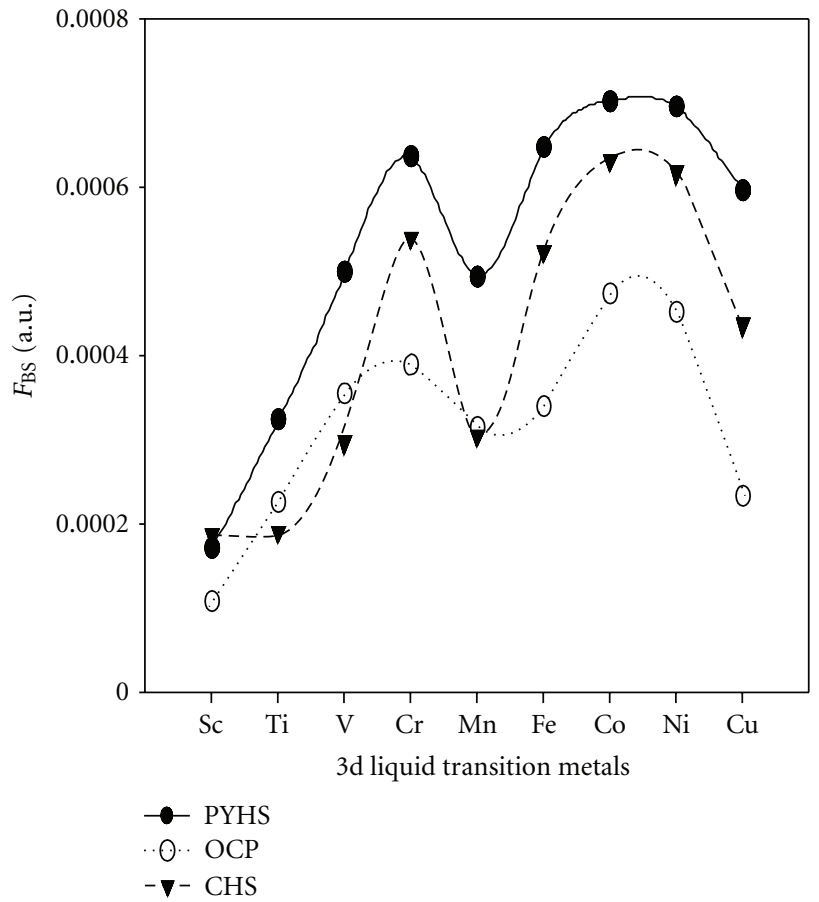

(a)

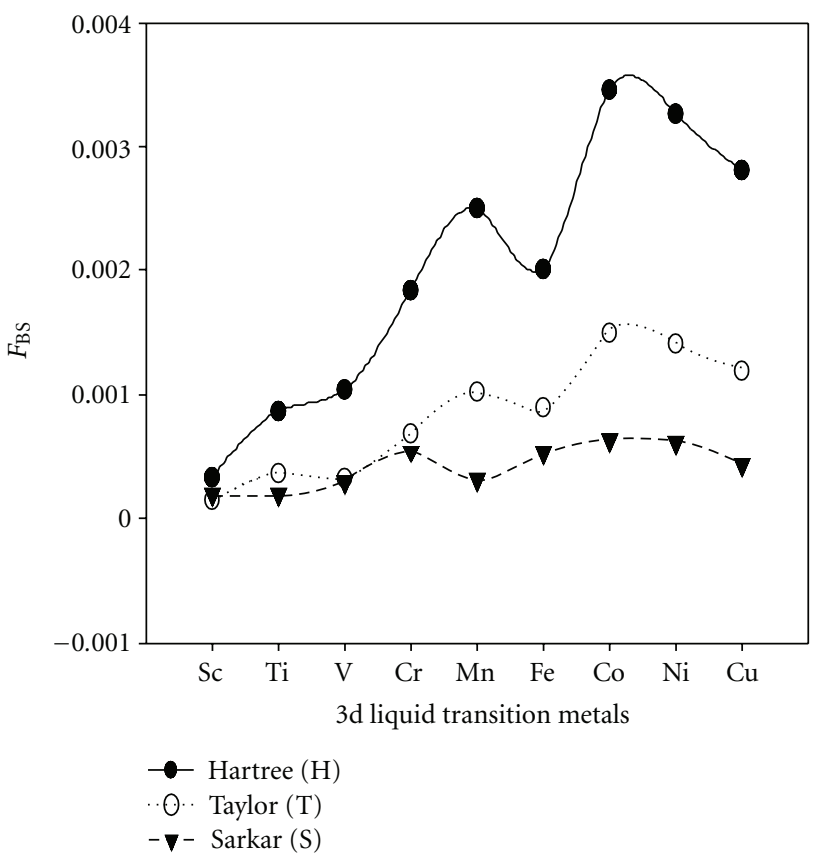

(b)

FIgURE 4: Calculated band structure energy $\left(F_{\mathrm{BS}}\right)$ of 3d liquid transition metals for different reference system with Sarkar et al. (SR) [65] local field correction function and for different local field correction functions along with charged hard sphere (CHS) reference system.

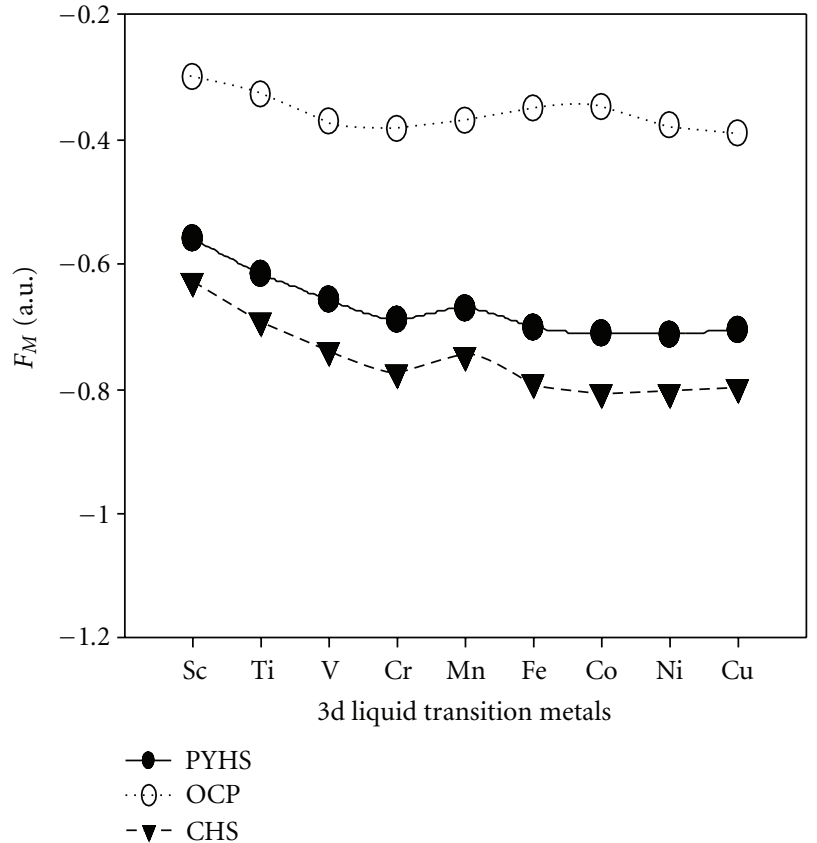

(a)

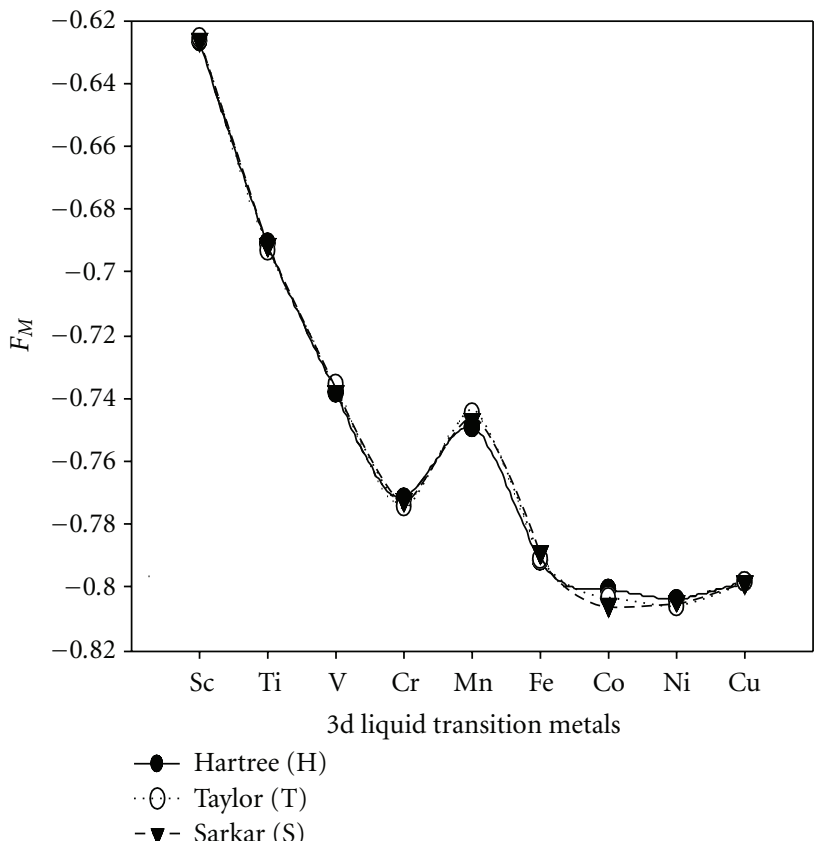

(b)

FIgure 5: Calculated Madelung energy $\left(F_{M}\right)$ of 3d liquid transition metals for different reference system with Sarkar et al. (SR) [65] local field correction function and for different local field correction functions along with charged hard sphere (CHS) reference system. 
TAble 3: Calculated internal energy (E) due to different local field correction functions like Hartree (HR) [63], Taylor (TR) [64], and Sarkar et al. (SR) [65] of 3d liquid transition metals.

\begin{tabular}{|c|c|c|c|c|c|}
\hline \multirow{2}{*}{ Metal } & \multirow{2}{*}{$S(q)$} & \multicolumn{4}{|c|}{ Internal energy $(E)$ in au } \\
\hline & & HR [63] & TR [64] & SR [65] & Other $[10,35]$ \\
\hline \multirow{3}{*}{ Sc } & PYHS & -0.4897 & -0.4887 & -0.4886 & \multirow{3}{*}{-0.7180} \\
\hline & OCP & -0.5433 & -0.5437 & -0.5436 & \\
\hline & CHS & -0.5570 & -0.5548 & -0.5580 & \\
\hline \multirow{3}{*}{$\mathrm{Ti}$} & PYHS & -0.5286 & -0.5299 & -0.5290 & \multirow{3}{*}{$-0.7247,-0.6593$} \\
\hline & OCP & -0.5906 & -0.5909 & -0.5906 & \\
\hline & CHS & -0.6053 & -0.6064 & -0.6063 & \\
\hline \multirow{3}{*}{$\mathrm{V}$} & PYHS & -0.5653 & -0.5645 & -0.5634 & \multirow{3}{*}{$-0.7638,-0.7193$} \\
\hline & OCP & -0.6268 & -0.6271 & -0.6267 & \\
\hline & CHS & -0.6458 & -0.6439 & -0.6445 & \\
\hline \multirow{3}{*}{$\mathrm{Cr}$} & PYHS & -0.5544 & -0.5570 & -0.5550 & \multirow{3}{*}{$-0.7841,-0.7162$} \\
\hline & $\mathrm{OCP}$ & -0.6219 & -0.6223 & -0.6221 & \\
\hline & CHS & -0.6397 & -0.6409 & -0.6408 & \\
\hline \multirow{3}{*}{$\mathrm{Mn}$} & PYHS & -0.4976 & -0.5010 & -0.4989 & \multirow{3}{*}{$-0.7305,-0.6717$} \\
\hline & OCP & -0.5699 & -0.5702 & -0.5700 & \\
\hline & CHS & -0.5790 & -0.5781 & -0.5772 & \\
\hline \multirow{3}{*}{$\mathrm{Fe}$} & PYHS & -0.5594 & -0.5627 & -0.5606 & \multirow{3}{*}{$-0.7284,-0.7088$} \\
\hline & OCP & -0.6313 & -0.6349 & -0.6324 & \\
\hline & CHS & -0.6501 & -0.6528 & -0.6508 & \\
\hline \multirow{3}{*}{ Co } & PYHS & -0.5138 & -0.5187 & -0.5156 & \multirow{3}{*}{$-0.6649,-0.7134$} \\
\hline & OCP & -0.4866 & -0.4865 & -0.4867 & \\
\hline & CHS & -0.6042 & -0.6114 & -0.6078 & \\
\hline \multirow{3}{*}{$\mathrm{Ni}$} & PYHS & -0.5241 & -0.5289 & -0.5261 & \multirow{3}{*}{$-0.7971,-0.7257,-0.812$} \\
\hline & OCP & -0.5983 & -0.6031 & -0.6001 & \\
\hline & CHS & -0.6190 & -0.6198 & -0.6200 & \\
\hline \multirow{3}{*}{$\mathrm{Cu}$} & PYHS & -0.5312 & -0.5353 & -0.5327 & \multirow{3}{*}{-0.3779} \\
\hline & OCP & -0.6088 & -0.6094 & -0.6091 & \\
\hline & CHS & -0.6273 & -0.6281 & -0.6281 & \\
\hline
\end{tabular}

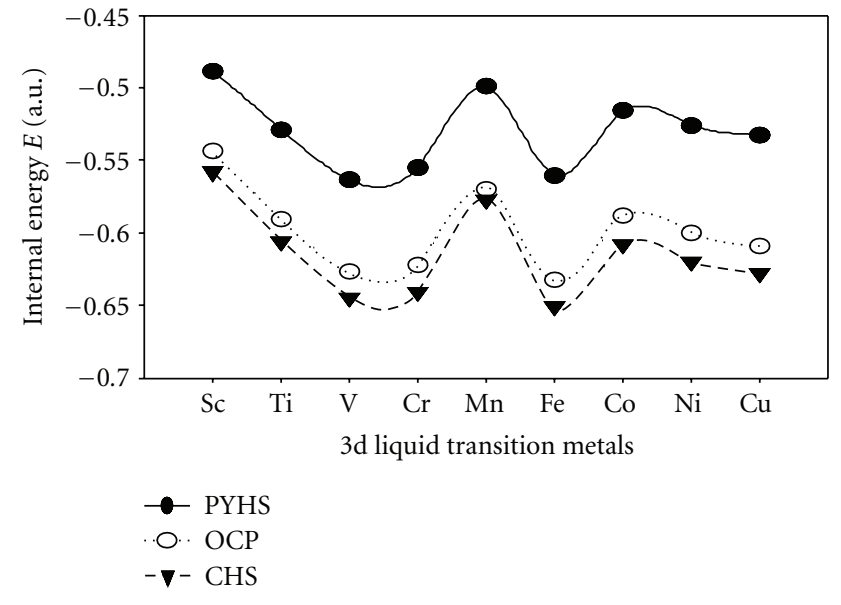

FIGURE 6: Calculated internal energy $(E)$ of 3d liquid transition metals for different reference system with Sarkar et al. (SR) [65] local field correction function. 
TABLE 4: Calculated entropy $\left(S / k_{B}\right)$ due to different local field correction functions like Hartree (HR) [63], Taylor (TR) [64], and Sarkar et al. (SR) [65] of liquid transition metals.

\begin{tabular}{|c|c|c|c|c|c|c|}
\hline \multirow{2}{*}{ Metal } & \multirow{2}{*}{$S(q)$} & \multicolumn{4}{|c|}{ Entropy $\left(S / k_{B}\right)$ in au } & \multirow[b]{2}{*}{ Expt. $[50,68]$} \\
\hline & & $\operatorname{HR}[63]$ & TR [64] & SR [65] & Other $[8,10,35,45,56]$ & \\
\hline \multirow{3}{*}{ Sc } & PYHS & 10.67 & 10.85 & 10.81 & \multirow{3}{*}{$11.48,11.56,11.87$} & \multirow{3}{*}{12.05} \\
\hline & OCP & 12.75 & 12.72 & 12.72 & & \\
\hline & CHS & 11.02 & 11.04 & 11.00 & & \\
\hline \multirow{3}{*}{$\mathrm{Ti}$} & PYHS & 10.68 & 10.67 & 10.68 & \multirow{3}{*}{$12.42,12.04,11.28,11.55,11.06$} & \multirow{3}{*}{12.01} \\
\hline & OCP & 12.44 & 12.41 & 12.41 & & \\
\hline & CHS & 10.86 & 10.85 & 10.85 & & \\
\hline \multirow{3}{*}{$\mathrm{V}$} & PYHS & 10.51 & 10.70 & 10.70 & \multirow{3}{*}{$12.15,12.06,11.35,11.61,11.24$} & \multirow{3}{*}{12.08} \\
\hline & $\mathrm{OCP}$ & 12.57 & 12.57 & 12.57 & & \\
\hline & CHS & 10.92 & 10.95 & 10.94 & & \\
\hline \multirow{3}{*}{$\mathrm{Cr}$} & PYHS & 10.45 & 10.47 & 10.47 & \multirow{3}{*}{$12.00,11.94,11.13,11.34,10.95$} & \multirow{3}{*}{11.58} \\
\hline & OCP & 12.38 & 12.41 & 12.38 & & \\
\hline & CHS & 10.74 & 10.73 & 10.73 & & \\
\hline \multirow{3}{*}{$\mathrm{Mn}$} & PYHS & 9.57 & 9.58 & 9.58 & \multirow{3}{*}{$11.14,11.37,10.65,10.87,10.41$} & \multirow{3}{*}{12.05} \\
\hline & OCP & 11.62 & 11.62 & 11.62 & & \\
\hline & CHS & 9.86 & 9.87 & 9.88 & & \\
\hline \multirow{3}{*}{$\mathrm{Fe}$} & PYHS & 9.95 & 9.94 & 9.94 & \multirow{3}{*}{$11.93,11.84,11.11,11.39,10.98$} & \multirow{3}{*}{12.11} \\
\hline & OCP & 11.90 & 11.88 & 11.90 & & \\
\hline & CHS & 10.14 & 10.15 & 10.14 & & \\
\hline \multirow{3}{*}{ Co } & PYHS & 9.99 & 9.98 & 10.00 & \multirow{3}{*}{$12.44,11.98,11.19,11.49,10.98$} & \multirow{3}{*}{12.08} \\
\hline & OCP & 12.09 & 12.12 & 12.09 & & \\
\hline & CHS & 10.17 & 10.14 & 10.14 & & \\
\hline \multirow{3}{*}{$\mathrm{Ni}$} & PYHS & 9.88 & 9.87 & 9.86 & \multirow{3}{*}{$12.76,12.06,11.27,11.56,11.08,11.21$} & \multirow{3}{*}{11.69} \\
\hline & $\mathrm{OCP}$ & 11.85 & 11.85 & 11.85 & & \\
\hline & CHS & 10.05 & 10.04 & 10.04 & & \\
\hline \multirow{3}{*}{$\mathrm{Cu}$} & PYHS & 9.30 & 9.31 & 9.32 & \multirow{3}{*}{$12.06,11.08,10.04,10.26,9.68,10.21$} & \multirow{3}{*}{10.27} \\
\hline & $\mathrm{OCP}$ & 11.44 & 11.44 & 11.44 & & \\
\hline & CHS & 9.45 & 9.45 & 9.45 & & \\
\hline
\end{tabular}

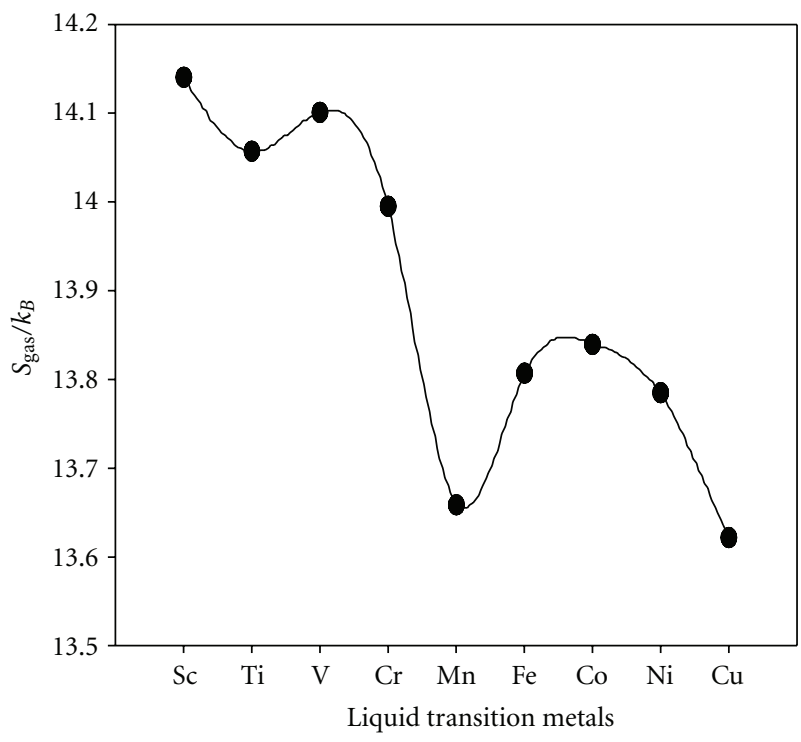

$3 d$ series

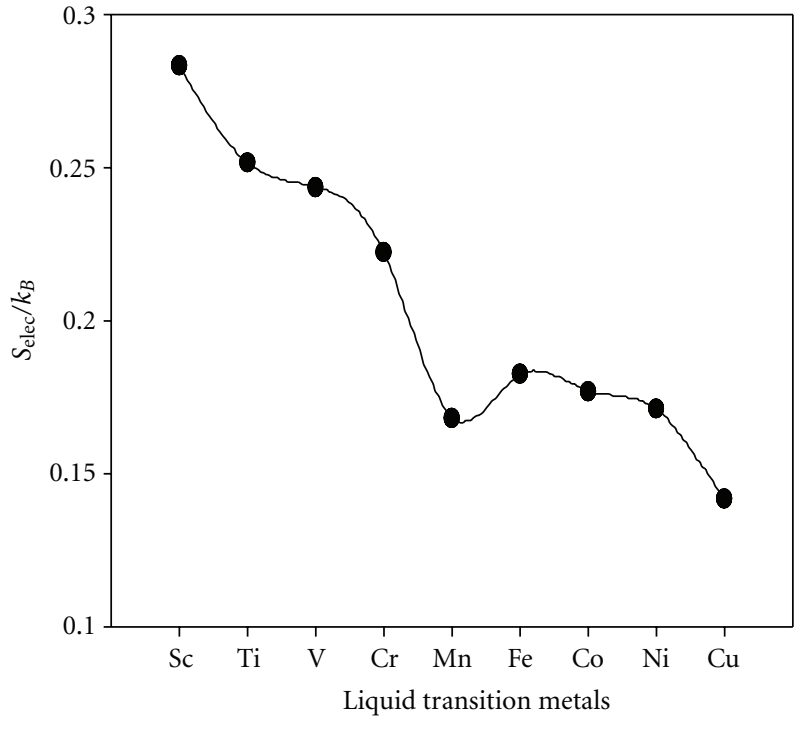

$3 \mathrm{~d}$ series

(a)

Figure 7: Calculated $S_{\text {gas }}$ and $S_{\text {elec }}$ of liquid transition metals. 
TABle 5: Calculated Helmholtz free energy $(F)$ due to different local field correction functions like Hartree (HR) [63], Taylor (TR) [64], and Sarkar et al. (SR) [65] of liquid transition metals.

\begin{tabular}{|c|c|c|c|c|c|}
\hline \multirow{2}{*}{ Metal } & \multirow{2}{*}{$S(q)$} & \multicolumn{4}{|c|}{ Helmholtz free energy $(F)$ in au } \\
\hline & & $\operatorname{HR}[63]$ & TR [64] & SR [65] & Others $[24,34,50]$ \\
\hline \multirow{3}{*}{ Sc } & PYHS & -0.6136 & -0.6145 & -0.6140 & \multirow{3}{*}{$-0.735,-0.755,-0.756$} \\
\hline & OCP & -0.6913 & -0.6914 & -0.6913 & \\
\hline & CHS & -0.6848 & -0.6830 & -0.6857 & \\
\hline \multirow{3}{*}{$\mathrm{Ti}$} & PYHS & -0.6620 & -0.6632 & -0.6624 & \multirow{3}{*}{$-0.704,-0.730,-0.734,-0.733,-0.731$} \\
\hline & OCP & -0.7460 & -0.7460 & -0.7457 & \\
\hline & CHS & -0.7409 & -0.7419 & -0.7418 & \\
\hline \multirow{3}{*}{$\mathrm{V}$} & PYHS & -0.7098 & -0.7117 & -0.7106 & \multirow{3}{*}{$-0.765,-0.798,-0.803,-0.768,-0.765$} \\
\hline & OCP & -0.7997 & -0.8000 & -0.7996 & \\
\hline & CHS & -0.7961 & -0.7945 & -0.7950 & \\
\hline \multirow{3}{*}{$\mathrm{Cr}$} & PYHS & -0.6982 & -0.7011 & -0.6992 & \multirow{3}{*}{$-0.757,-0.794,-0.799,-0.786,-0.783$} \\
\hline & OCP & -0.7922 & -0.7931 & -0.7924 & \\
\hline & CHS & -0.7875 & -0.7886 & -0.7885 & \\
\hline \multirow{3}{*}{$\mathrm{Mn}$} & PYHS & -0.5905 & -0.5940 & -0.5919 & \multirow{3}{*}{$-0.686,-0.721,-0.726,-0.736,-0.735$} \\
\hline & OCP & -0.6827 & -0.6830 & -0.6828 & \\
\hline & CHS & -0.6747 & -0.6739 & -0.6732 & \\
\hline \multirow{3}{*}{$\mathrm{Fe}$} & PYHS & -0.6749 & -0.6781 & -0.6760 & \multirow{3}{*}{$-0.743,-0.776,-0.778,-0.755,-0.754$} \\
\hline & OCP & -0.7695 & -0.7728 & -0.7706 & \\
\hline & CHS & -0.7678 & -0.7706 & -0.7684 & \\
\hline \multirow{3}{*}{ Co } & PYHS & -0.6290 & -0.6340 & -0.6310 & \multirow{3}{*}{$-0.752,-0.785,-0.785,-0.757,-0.756$} \\
\hline & OCP & -0.6262 & -0.6264 & -0.6263 & \\
\hline & CHS & -0.7215 & -0.7284 & -0.7249 & \\
\hline \multirow{3}{*}{$\mathrm{Ni}$} & PYHS & -0.6350 & -0.6397 & -0.6368 & \multirow{3}{*}{$-0.756,-0.790,-0.791,-0.751,-0.750$} \\
\hline & OCP & -0.7313 & -0.7361 & -0.7331 & \\
\hline & CHS & -0.7318 & -0.7325 & -0.7327 & \\
\hline \multirow{3}{*}{$\mathrm{Cu}$} & PYHS & -0.6150 & -0.6192 & -0.6166 & \multirow{3}{*}{$-0.746,-0.780,-0.783,-0.730,-0.726$} \\
\hline & OCP & -0.7119 & -0.7125 & -0.7121 & \\
\hline & CHS & -0.7125 & -0.7132 & -0.7132 & \\
\hline
\end{tabular}

and effect of local field correction functions on $3 \mathrm{~d}$ liquid transition metals for CHS system. The CHS system gives the lowest value of $F_{M}$ term while OCP system gives the highest values of $F_{M}$ term for liquid transition metals. From Figure 5, it is clear that $F_{M}$ term is not much sensitive to the choice of local field correction function. The calculated value of internal energy $(E)$ of $3 \mathrm{~d}$ liquid transition metals is tabulated in Table 3 along with other available data [10, 35] and also plotted in Figure 6 for Sarkar et al. (SR) [65] local field correction function. From these, it is clear that CHS system gives a lower internal energy $(E)$ compares to other systems for all liquid transition metals. It is also noticed that the value of internal energy has not been much affected by the influence of local field correction functions. The $F_{M}$ and $F_{\mathrm{EG}}$ give negative contribution to internal energy $(E)$ while $F_{1}$ and $F_{\mathrm{BS}}$ give positive contribution to internal energy $(E)$. $F_{1}$ term includes the electron-ion interaction through model potential. In this calculation major contribution comes from the term $F_{M}$ while contribution of $F_{\mathrm{BS}}$ is very small.

The calculated entropy of liquid transition metals is presented in Table 4. The minimum numerical values have been obtained due to the PYHS system while the maximum due to the OCP system. From the present results, we have observed that the OCP system gives excellent agreement with present results and experimental data $[50,68]$ for entropy compared to other reference system for $3 \mathrm{~d}$ liquid transition metals (except $\mathrm{Cu}$ ). A good agreement has been found with experimental data $[50,68]$ for liquid $\mathrm{Ti}, \mathrm{V}$, and Mn due to Sarkar et al. (SR) [65] local field correction function for OCP system while for liquid $\mathrm{Fe}, \mathrm{Co}$, and $\mathrm{Ni}$ due to Hartree (HR) [63] local field correction function for OCP system. The different contributions of entropy $(S)$, are plotted in Figures 7-9 along with Sarkar et al. (SR) [65] local field correction function of $3 \mathrm{~d}$ liquid transition metals. 
TABLE 6: Calculated long wave length limit of structure factor $S(0)$ due to different local field correction functions like Hartree (HR) [63], Taylor (TR) [64], and Sarkar et al. (SR) [65] of 3d liquid transition metals.

\begin{tabular}{|c|c|c|c|c|c|c|}
\hline \multirow{2}{*}{ Metal } & \multirow{2}{*}{$S(q)$} & \multicolumn{4}{|c|}{ Long wave length limit of structure factor $S(0)$} & \multirow[b]{2}{*}{ Expt. $[71,72]$} \\
\hline & & HR [63] & TR [64] & SR [65] & Other $[69,70]$ & \\
\hline \multirow{3}{*}{ Sc } & PYHS & 0.0280 & 0.0307 & 0.0300 & \multirow{3}{*}{ - } & \multirow{3}{*}{0.036} \\
\hline & $\mathrm{OCP}$ & 0.0252 & 0.0362 & 0.0340 & & \\
\hline & CHS & 0.0346 & 0.0349 & 0.0341 & & \\
\hline \multirow{3}{*}{$\mathrm{Ti}$} & PYHS & 0.0299 & 0.0297 & 0.0299 & \multirow{3}{*}{$0.0226,0.0236,0.0228$} & \multirow{3}{*}{0.020} \\
\hline & OCP & 0.0264 & 0.0386 & 0.0363 & & \\
\hline & CHS & 0.0337 & 0.0331 & 0.0332 & & \\
\hline \multirow{3}{*}{$\mathrm{V}$} & PYHS & 0.0268 & 0.0295 & 0.0295 & \multirow{3}{*}{$0.0234,0.0243,0.0236$} & \multirow{3}{*}{0.025} \\
\hline & OCP & 0.0283 & 0.0410 & 0.0387 & & \\
\hline & CHS & 0.0342 & 0.0344 & 0.0343 & & \\
\hline \multirow{3}{*}{$\mathrm{Cr}$} & PYHS & 0.0277 & 0.0280 & 0.0281 & \multirow{3}{*}{$0.0205,0.0212,0.0207$} & \multirow{3}{*}{0.021} \\
\hline & OCP & 0.0236 & 0.0316 & 0.0308 & & \\
\hline & CHS & 0.0329 & 0.0325 & 0.0325 & & \\
\hline \multirow{3}{*}{$\mathrm{Mn}$} & PYHS & 0.0217 & 0.0218 & 0.0218 & \multirow{3}{*}{$0.0231,0.0242,0.0233$} & \multirow{3}{*}{0.024} \\
\hline & OCP & 0.0136 & 0.0173 & 0.0167 & & \\
\hline & CHS & 0.0245 & 0.0244 & 0.0245 & & \\
\hline \multirow{3}{*}{$\mathrm{Fe}$} & PYHS & 0.0242 & 0.0240 & 0.0240 & \multirow{3}{*}{$0.0202,0.0210,0.0204$} & \multirow{3}{*}{0.020} \\
\hline & OCP & 0.0197 & 0.0273 & 0.0257 & & \\
\hline & CHS & 0.0265 & 0.0265 & 0.0264 & & \\
\hline \multirow{3}{*}{ Co } & PYHS & 0.0243 & 0.0242 & 0.0244 & \multirow{3}{*}{$0.0199,0.0207,0.0201$} & \multirow{3}{*}{0.019} \\
\hline & OCP & 0.0146 & 0.0180 & 0.0177 & & \\
\hline & CHS & 0.0264 & 0.0257 & 0.0259 & & \\
\hline \multirow{3}{*}{$\mathrm{Ni}$} & PYHS & 0.0237 & 0.0236 & 0.0234 & \multirow{3}{*}{$0.0199,0.0207,0.0201$} & \multirow{3}{*}{0.020} \\
\hline & OCP & 0.0154 & 0.0197 & 0.0191 & & \\
\hline & CHS & 0.0256 & 0.0253 & 0.0253 & & \\
\hline \multirow{3}{*}{$\mathrm{Cu}$} & PYHS & 0.0197 & 0.0198 & 0.0199 & \multirow{3}{*}{$0.0172,0.0179,0.0173$} & \multirow{3}{*}{0.018} \\
\hline & OCP & 0.0128 & 0.0165 & 0.0159 & & \\
\hline & CHS & 0.0207 & 0.0205 & 0.0205 & & \\
\hline
\end{tabular}

Among three contributions, only $S_{i}$ depends on structure and potential terms while $S_{\text {gas }}$ and $S_{\text {elec }}$ depend only on density and are independent of reference system and local field correction functions. The numerical value of $S_{\text {gas }}$ is much larger than $S_{i}$. The $S_{\text {gas }}$ increases the absolute value of entropy whereas $S_{i}$ tends to decrease the total entropy of system. From Figures 7-9, it is clear that PYHS system gives the lowest value of $S_{i}$ term while OCP system gives the highest numerical value of $S_{i}$ term of liquid transition metals. It is also noticed that $S_{i}$ term is not much sensitive to the use of different local field correction function. We have compared our results with available theoretical data $[10,35]$. The minimum numerical values of entropy $(S)$ have been obtained due to the PYHS system while the maximum due to the OCP system.

Table 5 represents Helmholtz free energy of 3d liquid transition metals. Figure 10 represents calculated Helmholtz free energy $F$ of $3 \mathrm{~d}$ liquid transition metals due to Sarkar et al.
(SR) [65] local field correction function. The OCP system gives minimum Helmholtz free energy compared to other reference system for liquid transition metals (except Co and $\mathrm{Cu}$ ). For $\mathrm{Co}$ and $\mathrm{Cu}$, the CHS system gives the minimum Helmholtz free energy. The values of Helmholtz free energy have not been much affected by the influence of local field correction functions. Hence we say that structure and potential are responsible to lower the free energy of the system. Therefore OCP and CHS systems show a lower Helmholtz free energy than PYHS. Among them OCP gives a lower Helmholtz free energy than other systems.

In our previous [60] attempt, we have thermodynamical properties like entropy $(S)$, internal energy $(E)$, and Helmholtz free energy $(F)$ of $3 \mathrm{~d}$ liquid transition metals using variational principle based on the Gibbs-Bogolyubov inequality with Percus-Yevick hard sphere reference system. But, here we have used three different reference systems 


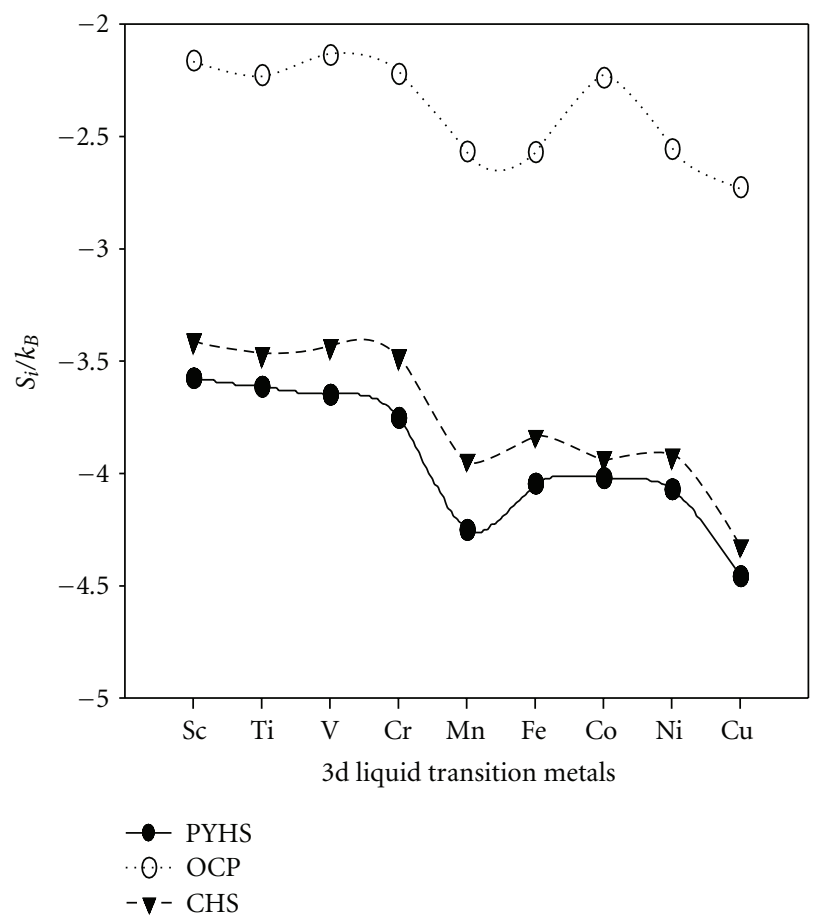

(a)

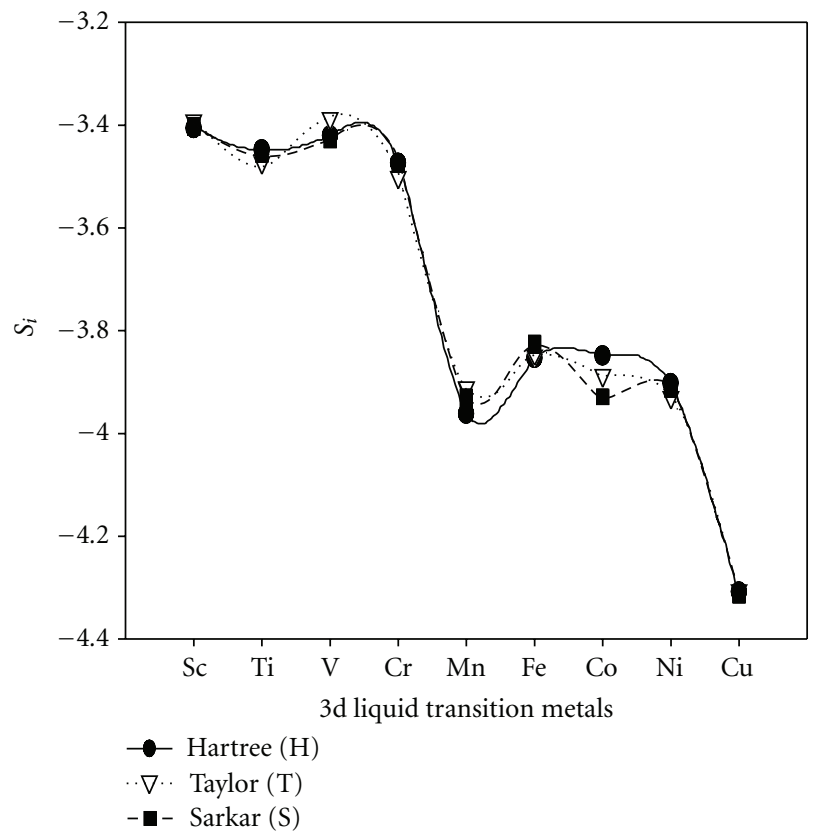

(b)

FIGURE 8: Calculated $S_{i}$ of 3d liquid transition metals for different reference systems with Sarkar et al. (SR) [65] local field correction function and for charged hard sphere (CHS) reference systems with different local field correction functions.

TABle 7: Calculated isothermal compressibility $\left(\chi_{T}\right)$ due to different local field correction functions like Hartree (HR) [63], Taylor (TR) [64], and Sarkar et al. (SR) [65] of 3d liquid transition metals.

\begin{tabular}{|c|c|c|c|c|c|c|}
\hline \multirow{2}{*}{ Metal } & \multirow{2}{*}{$S(q)$} & \multicolumn{4}{|c|}{ Isothermal compressibility $\chi_{T} \times 10^{-12} \mathrm{~cm}^{2} /$ dyne } & \multirow[b]{2}{*}{ Expt. $[45,71]$} \\
\hline & & HR [63] & TR [64] & SR [65] & Others [45] & \\
\hline \multirow{3}{*}{ Sc } & PYHS & 2.83 & 3.10 & 3.03 & \multirow{3}{*}{$2.25,2.31,1.91$} & \multirow{3}{*}{3.64} \\
\hline & OCP & 2.55 & 3.66 & 3.44 & & \\
\hline & CHS & 3.50 & 3.53 & 3.45 & & \\
\hline \multirow{3}{*}{$\mathrm{Ti}$} & PYHS & 2.10 & 2.09 & 2.11 & \multirow{3}{*}{$1.56,1.28$} & \multirow{3}{*}{$1.48,1.40$} \\
\hline & $\mathrm{OCP}$ & 1.86 & 2.72 & 2.56 & & \\
\hline & CHS & 2.37 & 2.33 & 2.34 & & \\
\hline \multirow{3}{*}{$\mathrm{V}$} & PYHS & 1.41 & 1.56 & 1.55 & \multirow{3}{*}{$1.25,1.23,1.01$} & \multirow{3}{*}{$1.10,1.31$} \\
\hline & OCP & 1.49 & 2.16 & 2.04 & & \\
\hline & CHS & 1.80 & 1.81 & 1.80 & & \\
\hline \multirow{3}{*}{$\mathrm{Cr}$} & PYHS & 1.27 & 1.29 & 1.29 & \multirow{3}{*}{$1.07,1.01,0.82$} & \multirow{3}{*}{$1.06,1.10$} \\
\hline & $\mathrm{OCP}$ & 1.09 & 1.45 & 1.41 & & \\
\hline & CHS & 1.51 & 1.49 & 1.49 & & \\
\hline \multirow{3}{*}{$\mathrm{Mn}$} & PYHS & 1.57 & 1.57 & 1.57 & \multirow{3}{*}{$1.63,1.52,1.23$} & \multirow{3}{*}{1.73} \\
\hline & $\mathrm{OCP}$ & 0.98 & 1.25 & 1.21 & & \\
\hline & CHS & 1.77 & 1.76 & 1.77 & & \\
\hline \multirow{3}{*}{$\mathrm{Fe}$} & PYHS & 1.26 & 1.26 & 1.26 & \multirow{3}{*}{$1.29,1.26,1.02$} & \multirow{3}{*}{$1.04,1.05$} \\
\hline & OCP & 1.03 & 1.43 & 1.34 & & \\
\hline & CHS & 1.38 & 1.38 & 1.38 & & \\
\hline \multirow{3}{*}{ Co } & PYHS & 1.23 & 1.23 & 1.23 & \multirow{3}{*}{$1.29,1.23,0.98$} & \multirow{3}{*}{$0.97,0.96$} \\
\hline & OCP & 0.74 & 0.91 & 0.89 & & \\
\hline & CHS & 1.33 & 1.30 & 1.31 & & \\
\hline \multirow{3}{*}{$\mathrm{Ni}$} & PYHS & 1.22 & 1.22 & 1.21 & \multirow{3}{*}{$1.21,1.26,1.00$} & \multirow{3}{*}{$0.98,1.03$} \\
\hline & $\mathrm{OCP}$ & 0.80 & 1.02 & 0.99 & & \\
\hline & CHS & 1.32 & 1.31 & 1.30 & & \\
\hline \multirow{3}{*}{$\mathrm{Cu}$} & PYHS & 1.33 & 1.34 & 1.34 & \multirow{3}{*}{1.58} & \multirow{3}{*}{$1.51,1.45$} \\
\hline & OCP & 0.86 & 1.11 & 1.07 & & \\
\hline & CHS & 1.39 & 1.38 & 1.38 & & \\
\hline
\end{tabular}


TABLE 8: Calculated specific heat $\left(C_{V} / k_{B}\right)$ due to different local field correction functions like Hartree (HR) [63], Taylor (TR) [64], and Sarkar et al. (SR) [65] of 3d liquid transition metals.

\begin{tabular}{|c|c|c|c|c|c|c|}
\hline \multirow{2}{*}{ Metal } & \multirow{2}{*}{$S(q)$} & \multicolumn{5}{|c|}{ Specific heat $\left(C_{V} / k_{B}\right)$} \\
\hline & & HR [63] & TR [64] & SR [65] & Others [45] & Expt. [45] \\
\hline \multirow{3}{*}{ Sc } & PYHS & 3.63 & 3.55 & 3.57 & \multirow{3}{*}{$3.72,3.91,4.75$} & \multirow{3}{*}{4.39} \\
\hline & OCP & 2.78 & 2.79 & 2.79 & & \\
\hline & CHS & 3.84 & 3.80 & 3.83 & & \\
\hline \multirow{3}{*}{$\mathrm{Ti}$} & PYHS & 3.57 & 3.58 & 3.57 & \multirow{3}{*}{$3.56,3.85,4.90$} & \multirow{3}{*}{3.53} \\
\hline & OCP & 2.77 & 2.79 & 2.79 & & \\
\hline & CHS & 3.86 & 3.84 & 3.85 & & \\
\hline \multirow{3}{*}{$\mathrm{V}$} & PYHS & 3.67 & 3.58 & 3.58 & \multirow{3}{*}{$3.59,3.87,5.17$} & \multirow{3}{*}{4.15} \\
\hline & OCP & 2.73 & 2.73 & 2.73 & & \\
\hline & CHS & 3.84 & 3.80 & 3.81 & & \\
\hline \multirow{3}{*}{$\mathrm{Cr}$} & PYHS & 3.64 & 3.63 & 3.62 & \multirow{3}{*}{$3.46,3.88,5.26$} & \multirow{3}{*}{3.90} \\
\hline & OCP & 2.74 & 2.72 & 2.74 & & \\
\hline & CHS & 3.85 & 3.84 & 3.84 & & \\
\hline \multirow{3}{*}{$\mathrm{Mn}$} & PYHS & 3.86 & 3.85 & 3.85 & \multirow{3}{*}{$3.27,3.75,4.33$} & \multirow{3}{*}{4.56} \\
\hline & OCP & 2.82 & 2.82 & 2.82 & & \\
\hline & CHS & 4.11 & 4.07 & 4.07 & & \\
\hline \multirow{3}{*}{$\mathrm{Fe}$} & PYHS & 3.76 & 3.76 & 3.76 & \multirow{3}{*}{$3.63,3.94,4.84$} & \multirow{3}{*}{4.38} \\
\hline & OCP & 2.79 & 2.80 & 2.79 & & \\
\hline & CHS & 4.09 & 4.06 & 4.07 & & \\
\hline \multirow{3}{*}{ Co } & PYHS & 3.75 & 3.75 & 3.75 & \multirow{3}{*}{$3.61 .4 .01,4.93$} & \multirow{3}{*}{4.00} \\
\hline & OCP & 2.72 & 2.71 & 2.72 & & \\
\hline & CHS & 4.07 & 4.07 & 4.07 & & \\
\hline \multirow{3}{*}{$\mathrm{Ni}$} & PYHS & 3.78 & 3.78 & 3.79 & \multirow{3}{*}{$3.76,4.13,4.99$} & \multirow{3}{*}{4.46} \\
\hline & OCP & 2.79 & 2.79 & 2.79 & & \\
\hline & CHS & 4.10 & 4.09 & 4.10 & & \\
\hline \multirow{3}{*}{$\mathrm{Cu}$} & PYHS & 3.95 & 3.95 & 3.94 & \multirow{3}{*}{$2.67,3.18,3.58$} & \multirow{3}{*}{3.04} \\
\hline & OCP & 2.84 & 2.84 & 2.84 & & \\
\hline & $\mathrm{CHS}$ & 4.36 & 4.35 & 4.36 & & \\
\hline
\end{tabular}

like Percus-Yevick Hard Sphere (PYHS) [23, 29-38], One Component Plasma (OCP) [37-44], and Charged Hard Sphere (CHS) [29, 30, 45-48]. Our present results for thermodynamical properties are improved compared to our previous attempt [60]. Thus in present investigation, we have seen the influence of structure factor $S(q)$ and different local field correction functions on thermodynamical and structural properties of $3 \mathrm{~d}$ liquid transition metals. It plays much more crucial role in the study of thermodynamical and structural properties of $3 \mathrm{~d}$ liquid transition metals compare to our previous studies [60].

The structure factor $S(q)$ obtained from the variational parameter $(\eta$ and $\Gamma)$ is illustrated in Figure 11. It is observed that in most of the cases overestimated the magnitude of the first peak, although in those cases the locations of the maxima and minima are reasonable predicted. We have explained the slight overestimation of our $S(q)$ value when compared with experiment in cases where they occur, as being that in those cases where $\eta$ is greater than 0.45 (which is the value expected from liquid state theory). The implication of this is that effective hard sphere diameter is too large and by interference, the repulsive part of the pair potential in polyvalent metals extends too far [30]. CHS systems gives better results for structure factor $S(q)$ compared to OCP system. OCP system gives much high peak in the structure factor compare to CHS system.

Table 6 represents calculated long wave length limit of structure factor $S(0)$ of 3 d liquid transition metals using our obtained variation parameters. The present results of long wave length limit $S(0)$ is also compared with available experimental $[71,72]$ as well as other data $[69,70]$. The present results are in a good agreement with available experimental data [71, 72] for $\mathrm{Ti}, \mathrm{V}, \mathrm{Cr}, \mathrm{Fe}$, and $\mathrm{Cu}$ liquid metals due to PYHS system, whereas for Sc and Mn due to CHS system and for $\mathrm{Co}$ and $\mathrm{Ni}$ liquid metals it is due to OCP system. Tables 7 and 8 show the calculated isothermal compressibility, $\left(\chi_{T}\right)$ and specific heat $\left(C_{V}\right)$ of $3 \mathrm{~d}$ liquid transition metals, respectively, along with available 


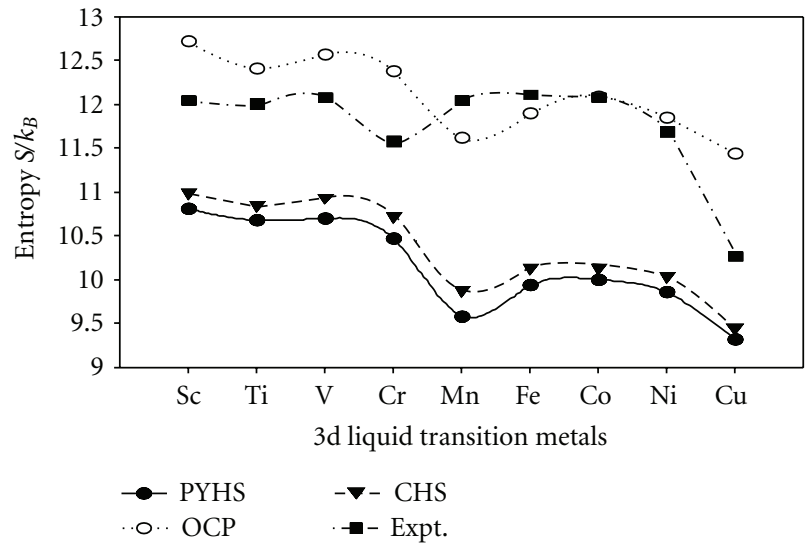

FIGURE 9: Calculated Entropy $(S)$ of 3d liquid transition metals for different reference systems with Sarkar et al. (SR) [65] local field correction function.

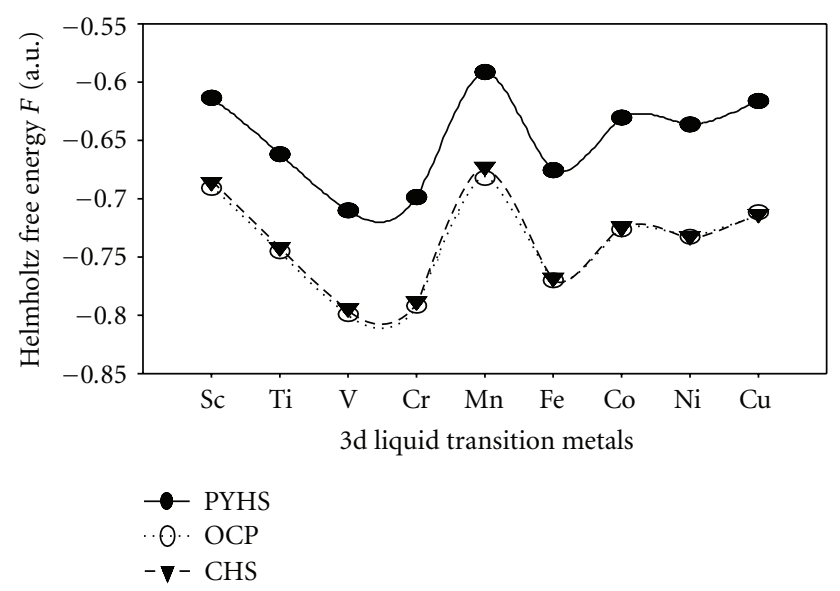

Figure 10: Calculated Hemlholtz free energy $(F)$ of 3d liquid transition metals for different reference systems with Sarkar et al. (SR) [65] local field correction function.

experimental data $[45,71]$ and other theoretical data [45]. The good agreement with available experimental data $[45$, 71] has been obtained for isothermal compressibility $\left(\chi_{T}\right)$ due to $\mathrm{CHS}$ for $\mathrm{Mn}$ and $\mathrm{Cu}$ liquid metals, OCP for Sc, Co, and Ni liquid metals and PYHS for $\mathrm{V}$ and Cr liquid metals. It is found that good agreement with available experimental data [45] have been achieved for specific heat $\left(C_{V}\right)$ of $\mathrm{Sc}, \mathrm{V}$, $\mathrm{Cr}, \mathrm{Fe}, \mathrm{Co}$, Ni liquid metals due to CHS system, whereas for $\mathrm{Ti}$ and $\mathrm{Cu}$ liquid metals it is due to PYHS and OCP systems respectively. So that overall CHS system is best suitable for calculating structural and thermodynamical properties of liquid transition metals along with our model potential compared to other reference system. This combination can be used to calculate other properties like electronic, magnetic, transport, and vibrational properties of liquid metals.

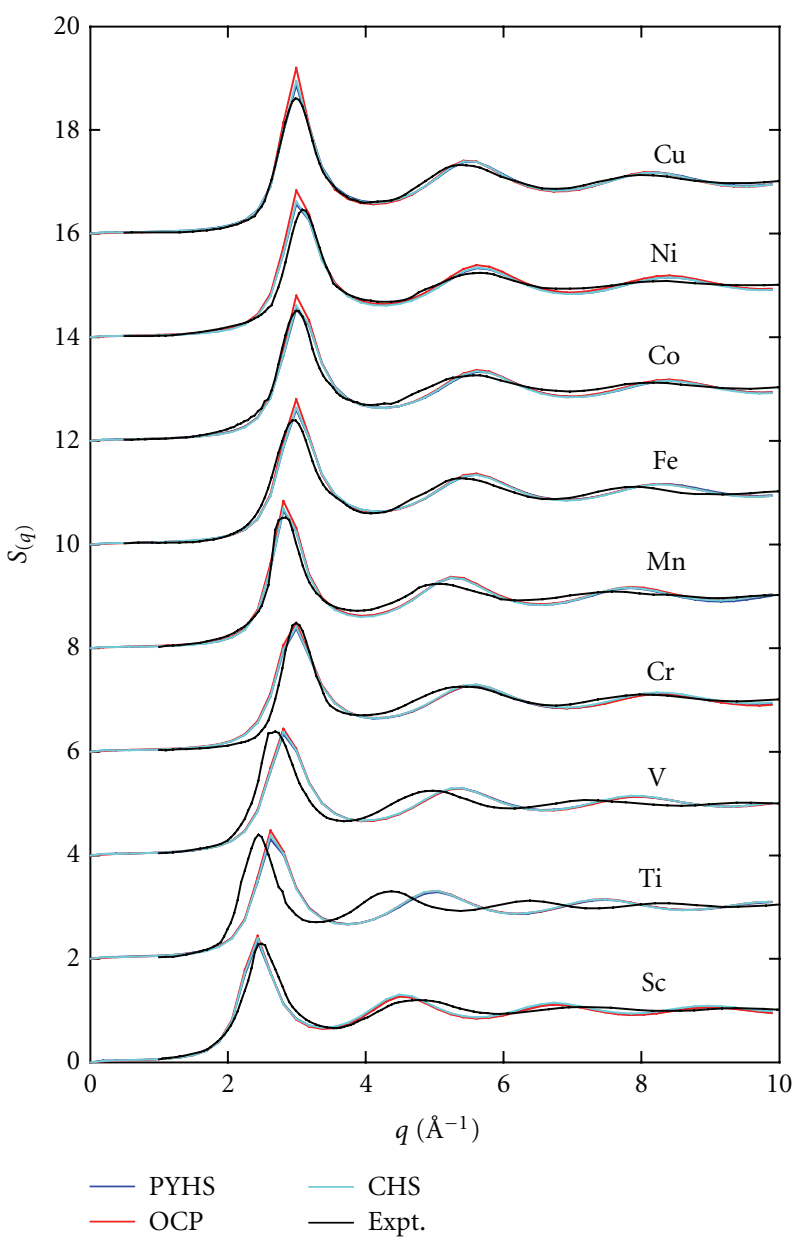

FIGURE 11: Structure factor $S(q)$ of $3 \mathrm{~d}$ liquid transition metals for Sarkar et al. (SR) [65] local field correction function.

\section{Summary and Conclusions}

(i) The thermodynamical and structural properties of liquid transition metals have been successfully investigated along with our newly constructed parameter free model potential.

(ii) These properties are very sensitive to the choice of reference systems. The good qualitative and quantitative data of the thermodynamical and structural properties of liquid transition metals have been obtained and would be beneficial to the other theoreticians as well as experimentalists working in the same field.

(iii) These properties of liquid metals are not sensitive to the use of the different local field correction functions.

(iv) Thus the application of our newly proposed model potential in the present study definitely adds new contribution to understand the thermodynamical and structural properties of transition metals in liquid state. 
(v) Thus the proper choice of the model potential along with the reference system plays a vital role in the study of the thermodynamical and structural properties of $3 \mathrm{~d}$ liquid transition metals.

\section{Acknowledgment}

P. B. Thakor and Y. A. Sonvane acknowledge the financial support from the University Grants Commission, New Delhi, India, under a Major Research Project F. no. 33-26/2007 (SR).

\section{References}

[1] J. M. Bomont, J. Delhommelle, and J. L. Bretonnet, "Structure and thermodynamics of the expanded liquid mercury by Monte Carlo simulation: a first attempt," Journal of NonCrystalline Solids, vol. 353, no. 32-40, pp. 3454-3458, 2007.

[2] E. Chacon, P. Tarazona, J. A. Verges, M. Reinaldo-Falagan, E. Velasco, and J. P. Hernandez, "Thermodynamic properties and electrical conductivity of a tight-binding hard-sphere model for liquid metals," Journal of Non-Crystalline Solids, vol. 353, pp. 3523-3527, 2007.

[3] N. E. Dubinin, L. D. Son, and N. A. Vatolin, "The WillsHarrison approach to the thermodynamics of binary liquid transition-metal alloys," Journal of Physics, vol. 20, no. 11, Article ID 114111, 2008.

[4] Y. N. Zhang, L. Wang, S. Morioka, and W. M. Wang, "Modified Lennard-Jones potentials for $\mathrm{Cu}$ and $\mathrm{Ag}$ based on the dense gaslike model of viscosity for liquid metals," Physical Review $B$, vol. 75, no. 1, Article ID 014106, 9 pages, 2007.

[5] A. Kumara, S. M. Rafiquea, N. Jhab, and A. K. Mishra, "Structure, thermodynamic, electrical and surface properties of $\mathrm{Cu}-\mathrm{Mg}$ binary alloy: complex formation model," Physica B, vol. 357, pp. 445-451, 2007.

[6] F. Sommer, "Thermodynamics of liquid alloys," Journal of Non-Crystalline Solids, vol. 353, no. 32-40, pp. 3709-3716, 2007.

[7] G. M. Bhuiyan, A. Rahman, M. A. Khaleque, R. I. M. A. Rashid, and S. M. M. Rahman, "Structural, thermodynamic and transport properties of liquid noble and transition metals," Physics and Chemistry of Liquids, vol. 38, no. 1, pp. $1-16,2000$.

[8] M. M. G. Alemany, M. Calleja, C. Rey, L. J. Gallego, J. Casas, and L. E. González, "Theoretical and computer simulation study of the static structure and thermodynamic properties of liquid transition metals using the embedded atom model," Journal of Non-Crystalline Solids, vol. 250, pp. 53-58, 1999.

[9] J. Z. Wang, M. Chen, and Z. Y. Guo, "Structural and thermodynamic properties of liquid transition metals with different embedded-atom method models," Chinese Physics Letters, vol. 19, no. 3, p. 324, 2002.

[10] N. Jakse, J. F. Wax, J. L. Bretonnet, and A. Pasturel, "Structure and thermodynamics of the 3d-transition metals in the liquid state," Journal of Non-Crystalline Solids, vol. 205-207, no. 1, pp. 434-437, 1996.

[11] M. Rasolt and R. Taylor, "Charge densities and interionic potentials in simple metals: nonlinear effects. I," Physical Review B, vol. 11, no. 8, pp. 2717-2725, 1975.

[12] L. Dagens, M. Rasolt, and R. Taylor, "Charge densities and interionic potentials in simple metals: nonlinear effects. II," Physical Review B, vol. 11, no. 8, pp. 2726-2734, 1975.
[13] G. Pastore and M. P. Tosi, "Structure factor of liquid alkali metals using a classical-plasma reference system," Physica B, vol. 124, no. 3, pp. 383-391, 1984.

[14] G. Kahl and J. Hafner, "Optimized random-phase approximation for the structure of expanded fluid rubidium," Physical Review A, vol. 29, no. 6, pp. 3310-3319, 1984.

[15] C. Regnaut, "Thermodynamics and structure of liquid metals: the variational approach versus the optimised random phase approximation," Journal of Physics F, vol. 16, no. 3, p. 295, 1986.

[16] K. Lad and A. Pratap, "Velocity autocorrelation function for simple liquids and its application to liquid metals and alloys," Physical Review E, vol. 70, no. 5, Article ID 051201, 9 pages, 2004.

[17] R. N. Singh, R. P. Jaju, and I. Ali, "Effective pair potential, structure and heat capacity of undercooled liquid rubidium," Physica B, vol. 299, no. 1-2, pp. 108-119, 2001.

[18] A. Mizuno, T. Masaki, and T. Itami, "Theoretical prediction of atomic volume for liquid metals based on the hard sphere model combined with NFE theory," Chemical Physics Letters, vol. 363, pp. 337-342, 2002.

[19] I. Ali, S. M. Osman, and R. N. Singh, "Compressibility factor for double Yukawa fluid," Journal of Non-Crystalline Solids, vol. 250-252, pp. 364-367, 1999.

[20] R. N. Singh and R. P. Jaju, "Energetics of undercooled liquid metals," Physica B, vol. 240, no. 1-2, pp. 133-141, 1997.

[21] S. Singh and R. N. Singh, "Effect of core-ion potentials on thermodynamic properties of liquid Alkali metals and alloys," Physics and Chemistry of Liquids, vol. 27, no. 4, pp. 203-214, 1994.

[22] O. Akinlade, L. A. Hussain, and F. Matthew, "The effect of local field corrections on the electrical resistivity of liquid metals," Physica Status Solidi B, vol. 169, no. 1, pp. 151-155, 1992.

[23] O. Akinlade, "Thermodynamics and structure of liquid metals from the soft sphere reference fluid," Physica Status Solidi B, vol. 169, no. 1, pp. 23-31, 1992.

[24] O. Akinlade, Z. Badirkhan, and G. Pastore, "Thermodynamics and structure of liquid metals from a new consistent optimized random phase approximation," Zeitschrift fur Naturforschung A, vol. 56, no. 9-10, pp. 605-612, 2001.

[25] D. H. Li, X. R. Li, and S. Wang, "Variational calculation of Helmholtz free energies with applications to the sp-type liquid metals," Journal of Physics F, vol. 16, no. 3, p. 309, 1986.

[26] S. K. Lai, M. Matsuura, and S. Wang, "Variational thermodynamic calculation for simple liquid metals and alkali alloys," Journal of Physics F, vol. 13, no. 10, p. 2033, 1983.

[27] D. Prato and D. E. Barraco, "Bogoliubov inequality," Revista Mexicana de Fisica, vol. 42, no. 1, pp. 145-150, 1996.

[28] N. N. Bogoliubov, "On the theory of superfluidity," Journal of Physics, vol. 11, p. 23, 1947.

[29] O. Akinlade, S. K. Lai, and M. P. Tosi, "Thermodynamics and structure of liquid metals from the charged hard-sphere reference fluid," Physica B, vol. 167, no. 1, pp. 61-70, 1990.

[30] O. Akinlade, "A study of the structure and thermodynamics of liquid metals based on some reference systems," Physica Status Solidi B, vol. 161, no. 1, pp. 75-83, 1990.

[31] S. Singh and R. N. Singh, "Effect of core-ion potentials on thermodynamic properties of liquid alkali metals and alloys," Physics and Chemistry of Liquids, vol. 27, no. 4, pp. 203-214, 1994.

[32] R. N. Singh and F. Sommer, "Thermodynamic and structural interpretations of the undercooled liquid metals," Physics and Chemistry of Liquids, vol. 28, no. 2, pp. 129-140, 1994. 
[33] J. L. Bretonnet and N. Jakse, "Use of integral-equation theory in determining the structure and thermodynamics of liquid alkali metals," Physical Review B, vol. 50, no. 5, pp. 2880-2888, 1994.

[34] J. L. Bretonnet and A. Derouiche, "Variational thermodynamic calculations for liquid transition metals," Physical Review B, vol. 43, no. 11, pp. 8924-8929, 1991.

[35] A. M. Vora, "Thermodynamics of liquid d- and f-shell metals: a variational approach," Physics and Chemistry of Liquids, vol. 46, no. 3, pp. 278-286, 2008.

[36] J. K. Baria, "Analysis of thermodynamics of liquid d- and fshell metals with the variational approach," Chinese Physics Letters, vol. 20, no. 6, p. 894, 2003.

[37] M. Iwamatsu, "Calculation of the free energy of liquid alkali metal using the non-local pseudopotential-the onecomponent-plasma and the hard-sphere reference systems," Physica B, vol. 138, no. 3, pp. 310-314, 1986.

[38] K. K. Mon, R. Gann, and D. Stroud, "Thermodynamics of liquid metals: the hard-sphere versus one-compoent-plasma reference systems," Physical Review A, vol. 24, no. 4, pp. 21452150, 1981.

[39] S. K. Lai, "Accurate calculation of the Helmholtz free energy for simple liquid metals," Physical Review A, vol. 38, no. 11, pp. 5707-5713, 1988.

[40] M. Iwamatsu, R. A. Moore, and S. Wang, "On the use of the one-component-plasma model for variational thermodynamic calculations," Physics Letters A, vol. 101, no. 2, pp. 9799, 1984.

[41] M. Hasegawa, "Thermodynamic perturbation approach to freezing of the classical one-component plasma," Journal of the Physical Society of Japan, vol. 64, pp. 4248-4257, 1995.

[42] N. H. March and J. A. Alonso, "Some properties of the structure factor $\mathrm{S}(\mathrm{q})$ in two-dimensional classical liquids near freezing," Physics and Chemistry of Liquids, vol. 48, no. 3, pp. 409-413, 2010.

[43] P. B. Thakor, Y. A. Sonvane, and A. R. Jani, "Structural properties of some liquid transition metals," Physics and Chemistry of Liquids, vol. 49, no. 4, pp. 530-549, 2011.

[44] T. Arai, S. Naito, and I. Yokoyama, "Effective valencies of liquid $3 \mathrm{~d}$ transition metals estimated from the gibbs-bogoliubov variational method using the one-component plasma reference system," High Temperature Material Processes, vol. 12, no. 3, pp. 117-126, 1993.

[45] R. N. Joarder and T. Das, "Thermodynamics of liquid metals based on charged hard sphere (CHS) model," Physica Scripta, vol. 37 , no. 5 , p. $762,1988$.

[46] M. Iwamatsu, "On the calculation of the thermodynamics of liquid metals using the charged-hard-sphere reference system," Physica B, vol. 159, no. 3, pp. 269-274, 1989.

[47] S. K. Lai, O. Akinlade, and M. P. Tosi, "Thermodynamics and structure of liquid alkali metals from the charged-hard-sphere reference fluid," Physical Review A, vol. 41, no. 10, pp. 54825490, 1990.

[48] T. Das and R. N. Joarder, "Orpa calculation of structure factors of liquid alkali metals using CHS as reference system," Physics Letters A, vol. 131, no. 2, pp. 111-114, 1988.

[49] C. Hausleitner and J. Hafner, "Soft-sphere reference system in thermodynamic variational calculations. I. Liquid simple metals," Journal of Physics F, vol. 18, no. 6, p. 1013, 1988.

[50] C. Hausleitner and J. Hafner, "Soft-sphere reference system in thermodynamic variational calculations. II. Liquid transition metals," Journal of Physics F, vol. 18, no. 6, p. 1025, 1988.
[51] M. H. Ghatee and M. Bahadori, "Inter-ionic potential function in liquid alkali metals by thermodynamic regularity approach," Chemical Physics, vol. 335, no. 2-3, pp. 215-220, 2007.

[52] H. C. Chen and S. K. Lai, "Structure and thermodynamics of liquid alkali metals in variational modified hypernetted-chain theory," Physical Review A, vol. 45, pp. 3831-3840, 1992.

[53] T. Arai, S. Naito, and I. Yokoyama, "Correlation entropies of the hard sphere fluid based on the generalized mean spherical approximation due to Waisman," Physica B, vol. 270, pp. 52$59,1999$.

[54] I. Yokoyama and S. Tsuchiya, "Correlation entropy and its relation to properties of simple liquid metals," Journal of NonCrystalline Solids, vol. 312-314, pp. 232-235, 2002.

[55] I. Yokoyama, "Relationship between structural, thermodynamic, transport and surface properties of liquid metals: a hard-sphere description," Physica B, vol. 291, no. 1-2, pp. 145$151,2000$.

[56] I. Yokoyama and S. Naito, "Entropies and specific heats of liquid transition metals: one-component plasma model," Physica B, vol. 154, no. 3, pp. 309-312, 1989.

[57] C. Regnault, "Analysis of the liquid structure of 3d transition metals from the Wills-Harrison model," Zeitschrift für Physik B, vol. 76, no. 2, pp. 179-184, 1989.

[58] C. Hausleitner, G. Kahl, and J. Hafner, "Liquid structure of transition metals: investigations using molecular dynamics and perturbation- and integral-equation techniques," Journal of Physics, vol. 3, no. 11, pp. 1589-1602, 1991.

[59] P. B. Thakor, Y. A. Sonvane, and A. R. Jani, "Electronic transport properties of some transition liquid metals," Physics and Chemistry of Liquids, vol. 47, no. 6, pp. 653-662, 2009.

[60] P. B. Thakor, Y. A. Sonvane, and A. R. Jani, "Thermodynamical properties of $3 \mathrm{~d}$ transition liquid metals," in Proceedings of the AIP Conference Proceedings, vol. 1249, pp. 157-160, October 2009.

[61] M. L. Cohen, "Predicting new solids and superconductors," Science, vol. 234, no. 4776, pp. 549-553, 1986.

[62] N. W. Ashcroft and J. Lenker, "Structure and resistivity of liquid metals," Physical Review, vol. 145, pp. 83-90, 1966.

[63] W. A. Harrison, Elementary Electronic Structure, World Scientific, Singapore, 1999.

[64] R. Taylor, "A simple, useful analytical form of the static electron gas dielectric function," Journal of Physics F, vol. 8, no. 8, p. 1699, 1978.

[65] A. Sarkar, D. Sen, S. Haldar, and D. Roy, "Static local field factor for dielectric screening function of electron gas at metallic and lower densities," Modern Physics Letters B, vol. 12, no. 16, p. 639, 1998.

[66] G. M. Bhuiayan, M. Silbert, and M. J. Stott, "Structure and thermodynamic properties of liquid transition metals: an embedded-atom-method approach," Physical Review B, vol. 53, no. 2, pp. 636-645, 1996.

[67] Y. Waseda, The Structure of Non-Crystalline Materials Liquid and Amorphous Solids, McGraw-Hill, New York, NY, USA, 1980.

[68] R. Hultgren, P. D. Desai, and D. T. Wagman, Selected Values of the Thermodynamic Properties of the Elements, ASM International, Materials Park, Ohio, USA, 1973.

[69] S. Naito, T. Arai, I. Yokoyama, and Y. Waseda, "The longWavelength limit of the structure factor of liquid $3 \mathrm{~d}$ transition metals using one-component plasma model," High Temperature Material Processes, vol. 8, no. 2, pp. 125-134, 1989. 
[70] N. H. March and J. A. Alonso, “Transcending Lindemann's predictions for the melting temperatures of metals and for the long-wavelength limit of their liquid structure factors," Philosophical Magazine Letters, vol. 89, no. 4, pp. 300-305, 2009.

[71] Y. Waseda and S. Ueno, "The structure factors of liquid metals in low wavevector region," Science Reports of the Research Institutes, Tohoku University A, vol. 34, p. 15, 1987.

[72] Y. Waseda, F. Takahashi, and K. Suzuki, "Apparatus for X-ray diffraction of liquid metals and several results," Science Reports of the Research Institutes Tohoku University A, vol. 23, p. 128, 1971.

[73] J. A. Moriarty, "Analytic representation of multi-ion interatomic potentials in transition metals," Physical Review B, vol. 42, pp. 1609-1628, 1990. 

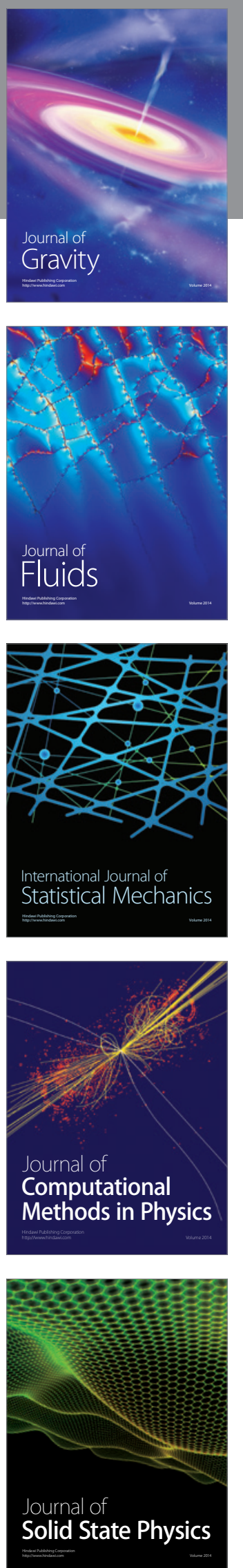
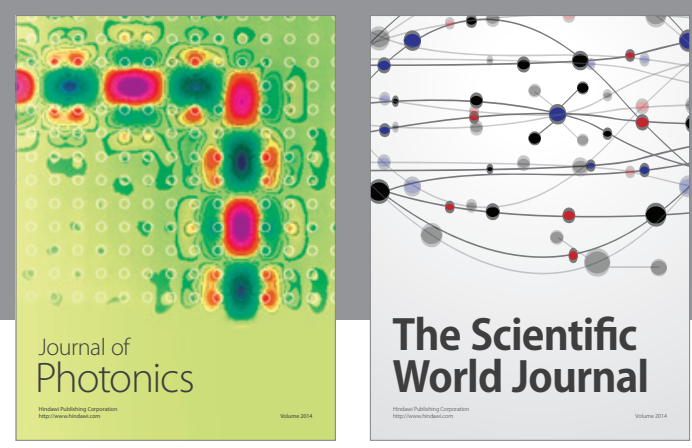

The Scientific World Journal

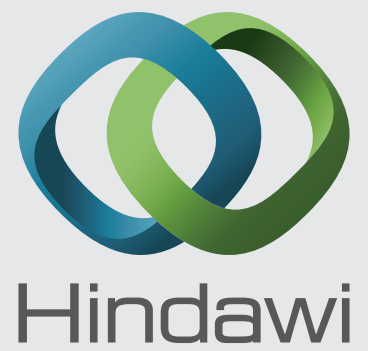

Submit your manuscripts at http://www.hindawi.com
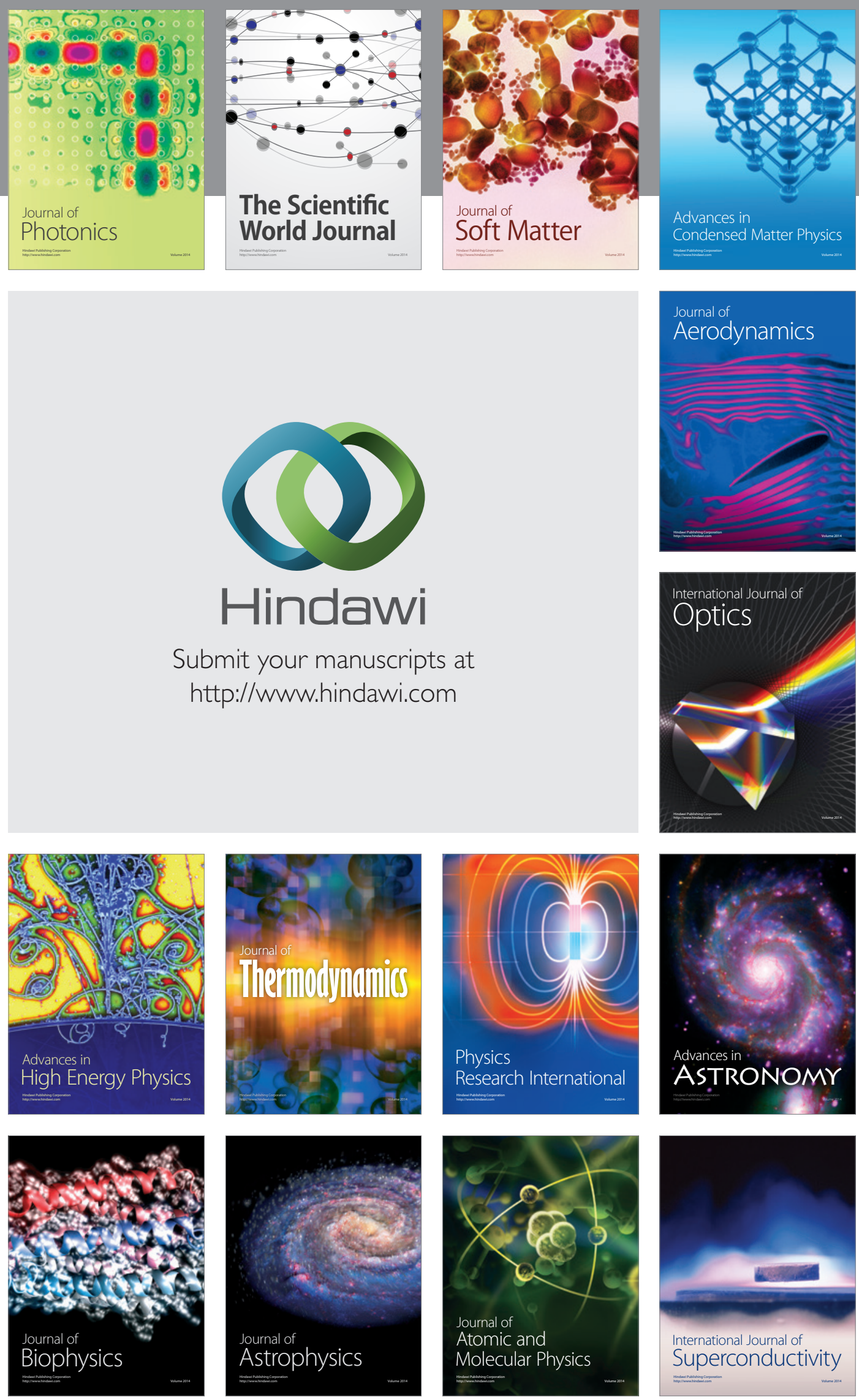
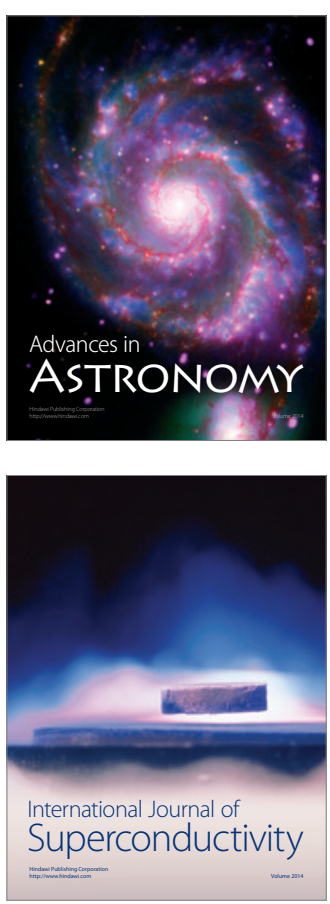